\title{
Energy and pressure versus volume: Equations of state motivated by the stabilized jellium model
}

\author{
Alim B. Alchagirov, ${ }^{1}$ John P. Perdew, ${ }^{1}$ Jonathan C. Boettger, ${ }^{2}$ R. C. Albers, ${ }^{2}$ and Carlos Fiolhais ${ }^{3}$ \\ ${ }^{1}$ Department of Physics and Quantum Theory Group, Tulane University, New Orleans, Louisiana 70118 \\ ${ }^{2}$ Theoretical Division, Los Alamos National Laboratory, Los Alamos, New Mexico 87545 \\ ${ }^{3}$ Center for Computational Physics, University of Coimbra, P3000 Coimbra, Portugal
}

(Received 13 September 2000; published 24 May 2001)

\begin{abstract}
Explicit functions are widely used to interpolate, extrapolate, and differentiate theoretical or experimental data on the equation of state (EOS) of a solid. We present two EOS functions which are theoretically motivated. The simplest realistic model for a simple metal, the stabilized jellium (SJ) or structureless pseudopotential model, is the paradigm for our SJEOS. A simple metal with exponentially overlapped ion cores is the paradigm for an augmented version (ASJEOS) of the SJEOS. For the three solids tested (Al, Li, Mo), the ASJEOS matches all-electron calculations better than prior equations of state. Like most of the prior EOS's, the ASJEOS predicts pressure $P$ as a function of compressed volume $v$ from only a few equilibrium inputs: the volume $v_{0}$, the bulk modulus $B_{0}$, and its pressure derivative $B_{1}$. Under expansion, the cohesive energy serves as another input. A further advantage of the new equation of state is that these equilibrium properties other than $v_{0}$ may be found by linear fitting methods. The SJEOS can be used to correct $B_{0}$ and the EOS found from an approximate density functional, if the corresponding error in $v_{0}$ is known. We also (a) estimate the typically small contribution of phonon zero-point vibration to the EOS, (b) find that the physical hardness $B v$ does not maximize at equilibrium, and (c) show that the "ideal metal" of Shore and Rose is the zero-valence limit of stabilized jellium.
\end{abstract}

DOI: 10.1103/PhysRevB.63.224115

PACS number(s): 64.10.+h, 64.30.+t, 62.20.Dc

\section{MOTIVATION}

The cohesion of a solid is reflected in its equation of state ${ }^{1}$ (EOS), i.e., in the dependence of its total energy or pressure upon its volume. Here we shall be concerned with the EOS for a solid constrained to a given crystal structure and to the paramagnetic state. Typically, parts of this EOS can be measured and all of it can be calculated approximately. The results are in both cases numerical, but can be fitted to an analytic form which can then be used to differentiate, interpolate, or extrapolate the data.

We review several widely used analytic forms for the EOS in Sec. II. All of them are phenomenological. As an alternative, we then propose in Secs. III and IV analytic forms that are based upon a microscopic model of cohesion. Even for the simplest model, the EOS is not exactly of simple analytic form, but it is nearly so as we shall see. The simple stabilized jellium model ${ }^{2}$ that we use to motivate our forms is rather realistic for the simple metals (certainly more so than the ordinary jellium model), but not so realistic for other solids. Nevertheless, the analytic forms it suggests could have a wider range of validity, comparable to or greater than that of the phenomenological forms. Moreover, our underlying microscopic model helps us to understand how to correct the EOS for errors in the calculated equilibrium lattice constant (Sec. VI).

For a constrained solid, the EOS should be reasonably smooth and continuous. We therefore follow a standard approach to approximate continuous functions: we try to build in as much of the correct asymptotics as we can. Thus, in our augmented stabilized jellium equation of state (ASJEOS of Sec. IV), we modify the stabilized jellium EOS to take account of core overlap under extreme compression, and of atom formation under extreme expansion. To describe the latter limit correctly, we must use the cohesive energy as another input to the EOS, in addition to the usual equilibrium volume, bulk modulus, and pressure derivative of bulk modulus at equilibrium.

For three metals (the simple metals $\mathrm{Al}$ and $\mathrm{Li}$, and the transition metal Mo), we will show by comparison with calculated energies and pressures that our ASJEOS works better than previous analytic forms like the Murnaghan ${ }^{3}$ EOS and the universal bonding energy relation ${ }^{4}$ (UBER). Under expansion, the ASJEOS works much better than either. Under compression, the ASJEOS works much better than the Murnaghan equation. For the stabilized-jellium-like metal Li under pressure, the ASJEOS also works much better than the UBER; for $\mathrm{Al}$ under pressure, it seems to work a little better than the UBER. However, we note that the UBER has been extensively tested and confirmed ${ }^{4-9}$ for a wide variety of solids under pressure, while our ASJEOS remains to be so widely tested. For greater accuracy the Murnaghan equation, which is still often used to estimate bulk moduli and their pressure derivatives, should be replaced by one of the more sophisticated forms.

Finally, we want to remark that our fitting (Sec. V) to the calculated energies is done only over a very narrow range of volumes around equilibrium, in order to extract the true values for the volume, bulk modulus, and pressure derivative of the bulk modulus at equilibrium. Fitting over a larger range of volumes would improve the apparent quality of the fit over the larger range by degrading our values for the equilibrium properties, and so would not rigorously test the various analytic forms for the EOS. We also discuss how to optimize the fitting range. Of course, we include volumes on both sides of equilibrium, because two-sided numerical differentiation is more accurate than one-sided differentiation. 


\section{INTRODUCTION TO THE EQUATION OF STATE}

The cold equation of state is a pressure-volume or energyvolume relation describing the behavior of a solid under compression and expansion at zero absolute temperature. It plays an important role in many fields, such as the physics of condensed matter or geophysics. Diamond-anvil cells can achieve static pressures up to $5 \mathrm{Mbar}$ or $500 \mathrm{GPa}$, while nuclear explosions can achieve dynamic pressures up to 10 Mbar (1 TPa) or more. ${ }^{1,10}$ The pressure in the Earth's inner core is about 4 Mbar. ${ }^{11}$

It is convenient to treat the EOS in terms of dimensionless parameters. Therefore we introduce the compression ratio or scale factor, defined as

$$
x=\left(\frac{v}{v_{0}}\right)^{1 / 3}
$$

where $v$ is the volume per unit cell (monatomic in our applications) and $v_{0}$ is its equilibrium value. Other quantities used in this paper are the bonding energy per unit cell $\varepsilon$ (which by definition tends to zero as $x \rightarrow \infty$ ), the pressure $P$, the bulk modulus or inverse compressibility $B$, and the first derivative of the bulk modulus with respect to pressure $B^{\prime}$ :

$$
\begin{gathered}
P=-\frac{d \varepsilon}{d v}=-\frac{1}{3 v_{0} x^{2}} \frac{d \varepsilon}{d x}, \\
B=-v \frac{d P}{d v}=-\frac{x}{3} \frac{d P}{d x}, \\
B^{\prime}=\frac{d B}{d P}=-\frac{x}{3} \frac{1}{B} \frac{d B}{d x},
\end{gathered}
$$

as well as their equilibrium values $\varepsilon_{0}=-\varepsilon(x=1)>0, P_{0}$ $=P(x=1)=0, B_{0}=B(x=1)$, and $B_{1}=B^{\prime}(x=1)$.

The EOS is often expressed as an analytic function $\varepsilon(x)$ or $P(x)$. One way the EOS can be used is to extract the bulk modulus $B_{0}$ and its first derivative $B_{1}$ at equilibrium by fitting to theoretical or experimental data. Another common application is to predict the high-pressure behavior of a solid from its low-pressure behavior or equilibrium properties. Both these applications are discussed in detail in this paper.

For a given volume $v$, the equilibrium crystal structure minimizes $\varepsilon$; for a given pressure $P$, it minimizes the enthalpy $h=\varepsilon+P v$. When all fundamental interactions are Coulombic, the virial theorem yields the kinetic energy contribution to $\varepsilon$ as $t=-\varepsilon+3 P v=t_{s}+t_{c}$ (Ref. 12), where $t_{s}$ and $t_{c}$ are the noninteracting and correlation contributions.

There are many equations of state in use. Some are constructed to describe specific crystal structures or materials. However, our major concern is the so-called "universal", equations of state. They have a universal form irrespective of the material, with a set of parameters specific for each one. One of the earliest and perhaps the best known of the equations of state is credited to Murnaghan: ${ }^{3}$

$$
\text { Murnaghan: } \quad P=\frac{B_{0}}{B_{1}}\left[x^{-3 B_{1}}-1\right] \text {, }
$$

which follows from the truncated expansion $B=B_{0}+B_{1} P$. Equation (5) can be analytically inverted from $P(x)$ to $x(P)=\left[1+B_{1}\left(P / B_{0}\right)\right]^{-1 /\left(3 B_{1}\right)}$. It is often used for $0<P$ $<B_{0} / 2$. Later $\operatorname{Birch}^{13,14}$ constructed what has become a widely used equation of state:

$$
\text { Birch: } \quad P=\frac{3}{2} B_{0}\left(x^{-7}-x^{-5}\right)\left[1+\frac{3}{4}\left(B_{1}-4\right)\left(x^{-2}-1\right)\right] \text {. }
$$

Another popular EOS, called the universal bonding energy relation, was advocated by Vinet and co-workers: ${ }^{4-6}$

$$
\text { UBER: } \quad P=3 B_{0} \frac{1-x}{x^{2}} e^{3\left(B_{1}-1\right)(1-x) / 2} \text {, }
$$

which happens to be exact for a harmonic crystal $\left(B_{1}=1\right)$. The UBER follows from the simple bonding energy formula

$$
\text { UBER: } \begin{aligned}
\varepsilon(x)= & -\frac{4 B_{0} v_{0}}{\left(B_{1}-1\right)^{2}}\left[1-\frac{3}{2}\left(B_{1}-1\right)\right. \\
& \times(1-x)] e^{3\left(B_{1}-1\right)(1-x) / 2} .
\end{aligned}
$$

It was found to be more realistic than the Murnaghan and Birch forms, especially at large compressions $\left(P \gg B_{0} / 2\right)$. The UBER was also applied successfully to a variety of different materials, including metallic, ionic, covalent, and noble-gas solids. When $P=B_{0}$, the UBER shows that $x$ is 0.85 for $B_{1}=3$ and 0.89 for $B_{1}=6$. The " $\mathrm{H} 02$ "' and " $\mathrm{H} 12$ ", equations of state proposed by Holzapfel $^{1}$ are designed to describe extreme compression $(x \ll 1)$, where the UBER must eventually fail:

$$
\begin{aligned}
& \text { H02: } P=3 B_{0} x^{-5}(1-x) e^{3\left(B_{1}-3\right)(1-x) / 2}, \\
& \text { H12: } P= \\
& \quad 3 B_{0} x^{-5}(1-x) e^{c_{0}(1-x)} \\
& \times e^{\left[3\left(B_{1}-3\right) / 2-c_{0}\right] x(1-x)},
\end{aligned}
$$

where $c_{0}=-\ln \left(3 B_{0} / P_{F G}\right)$, and

$$
P_{F G}=\frac{\left(3 \pi^{2}\right)^{2 / 3}}{5} \frac{\hbar^{2}}{m_{e}}\left(\frac{Z}{v_{0}}\right)^{5 / 3}
$$

is the free-electron Fermi-gas pressure at $x=1 ; Z$ is the total number of core and valence electrons in volume $v_{0}$, i.e., the nuclear charge. With the exception of $\mathrm{H} 12$, these expressions for $P(x) / B_{0}$ depend upon only one material parameter $\left(B_{1}\right)$ and may be integrated analytically to find $\varepsilon(x)$. Typically $3 \lesssim B_{1} \lesssim 6$ for metals.

All the present equations of state face common problems. First, they are largely semiempirical, lacking a microscopic foundation based upon insight into cohesion. Second, none of those described above has the cohesive energy $\varepsilon_{0}$ as a parameter, and they often fail to yield a realistic cohesive energy. To compute $\varepsilon_{0}$ from $P(x)$, one needs the definite integral 
TABLE I. Cohesive energies $\varepsilon_{0}$ from various equations of state [Eqs. (13)-(15)]. For simple metals we have used experimental values for the equilibrium volume $v_{0}$, the bulk modulus $B_{0}$, and its pressure derivative $B_{1}$ from Ref. 63. For Mo, the experimental values for $v_{0}$ and $B_{0}$ are from Ref. 74, and the experimental $B_{1}$ is from Ref. 75. Experimental values for $\varepsilon_{0}$ are from Ref. 74. The simple metals are presented in increasing order of $B_{1}$, making it clear that the experimental $\varepsilon_{0} /\left(B_{0} v_{0}\right)$ is not a function of $B_{1}$ alone. Note that $r_{0}=z^{1 / 3} r_{s}=\left(3 v_{0} /(4 \pi)\right)^{1 / 3}$. (1 hartree/bohr ${ }^{3}=294.2$ Mbar.) Also shown is $F=2 \sqrt{-2 \varepsilon_{H O}^{L D A}} r_{0}$ for use in Eq. (41), with $\varepsilon_{H O}^{L D A}$ from Ref. 47.

\begin{tabular}{|c|c|c|c|c|c|c|c|c|c|}
\hline \multirow[t]{2}{*}{ Metal } & \multirow[t]{2}{*}{$z$} & \multirow{2}{*}{$\begin{array}{c}r_{0}^{\text {expt }} \\
\text { (bohr) }\end{array}$} & \multirow{2}{*}{$\begin{array}{c}B_{0}^{\text {expt }} \\
\text { (Mbar) }\end{array}$} & \multirow[t]{2}{*}{$B_{1}^{\text {expt }}$} & \multicolumn{4}{|c|}{$\varepsilon_{0} / B_{0} v_{0}$} & \multirow[t]{2}{*}{$F$} \\
\hline & & & & & Expt. & Birch & UBER & $\mathrm{H} 02$ & \\
\hline $\mathrm{Ca}$ & 2 & 4.12 & 0.152 & 3.2 & 0.45 & 1.58 & 0.83 & 2.05 & 4.38 \\
\hline $\mathrm{Ba}$ & 2 & 4.67 & 0.103 & 3.4 & 0.47 & 1.46 & 0.69 & 1.39 & 4.56 \\
\hline $\mathrm{Sr}$ & 2 & 4.50 & 0.116 & 3.5 & 0.42 & 1.41 & 0.64 & 1.19 & 4.62 \\
\hline $\mathrm{Li}$ & 1 & 3.24 & 0.133 & 3.5 & 0.93 & 1.41 & 0.64 & 1.19 & 2.98 \\
\hline $\mathrm{Mg}$ & 2 & 3.34 & 0.369 & 3.9 & 0.28 & 1.18 & 0.48 & 0.73 & 3.96 \\
\hline $\mathrm{Na}$ & 1 & 3.93 & 0.073 & 3.9 & 0.65 & 1.18 & 0.48 & 0.73 & 3.57 \\
\hline Cs & 1 & 5.62 & 0.023 & 4.0 & 0.51 & 1.13 & 0.44 & 0.66 & 4.47 \\
\hline K & 1 & 4.86 & 0.037 & 4.1 & 0.57 & 1.07 & 0.42 & 0.60 & 4.10 \\
\hline $\mathrm{Rb}$ & 1 & 5.20 & 0.029 & 4.1 & 0.54 & 1.07 & 0.42 & 0.60 & 4.30 \\
\hline $\mathrm{Ga}$ & 3 & 3.16 & 0.568 & 4.1 & 0.40 & 1.07 & 0.42 & 0.60 & 4.11 \\
\hline $\mathrm{Be}$ & 2 & 2.36 & 1.144 & 4.6 & 0.57 & 0.79 & 0.31 & 0.40 & 3.03 \\
\hline $\mathrm{Al}$ & 3 & 2.99 & 0.794 & 4.7 & 0.41 & 0.73 & 0.29 & 0.38 & 2.71 \\
\hline In & 3 & 3.48 & 0.418 & 4.8 & 0.37 & 0.68 & 0.28 & 0.35 & 3.14 \\
\hline $\mathrm{Pb}$ & 4 & 3.65 & 0.488 & 5.5 & 0.22 & 0.28 & 0.20 & 0.23 & 3.88 \\
\hline $\mathrm{Tl}$ & 3 & 3.58 & 0.382 & 5.7 & 0.28 & 0.17 & 0.18 & 0.21 & 3.22 \\
\hline $\mathrm{Sn}$ & 4 & 3.52 & 0.541 & 6.0 & 0.34 & 0.00 & 0.16 & 0.18 & 3.78 \\
\hline Mo & 6 & 2.93 & 2.73 & 4.7 & 0.26 & 0.73 & 0.29 & 0.38 & 3.19 \\
\hline
\end{tabular}

$\varepsilon_{0}=\varepsilon(x=\infty)-\varepsilon(x=1)=\int_{1}^{\infty} d x \frac{d \varepsilon}{d x}=-3 v_{0} \int_{1}^{\infty} d x x^{2} P(x)$.

The cohesive energies as functions of $B_{0}$ and $B_{1}$ are therefore

$$
\text { Murnaghan: } \varepsilon_{0}=+\infty \text {, }
$$

Birch: $\quad \varepsilon_{0}=B_{0} v_{0} \frac{9}{16}\left(6-B_{1}\right)$,

$$
\text { UBER: } \quad \varepsilon_{0}=B_{0} v_{0} \frac{4}{\left(B_{1}-1\right)^{2}} \text {, }
$$

$$
\text { H02: } \varepsilon_{0}=B_{0} v_{0}\left[\frac{9}{2}+\frac{9}{2} c-9\left(c+\frac{c^{2}}{2}\right) e^{c} \int_{c}^{\infty} d y \frac{e^{-y}}{y}\right] \text {, }
$$

where $c=\frac{3}{2}\left(B_{1}-3\right)$. These integrals computed for various metals are presented in Table I. This table shows no particular tendency for the experimental $\varepsilon_{0} /\left(B_{0} v_{0}\right)$ to be a function of $B_{1}$ alone, unless we omit the divalent and tetravalent metals. Of course, no one actually uses the cohesive energies of Eqs. (12)-(15), but we present them to show that the standard equations of state are all seriously wrong on the expansion side of equilibrium.
We suggest that at least four parameters are required to describe the energy EOS properly: the equilibrium volume $v_{0}$ describes the volume at the energy minimum, the equilibrium cohesive energy $\varepsilon_{0}$ describes the depth of the EOS curve at equilibrium, and $B_{0}$ and $B_{1}$ describe the shape of the EOS at equilibrium:

$$
\varepsilon(x)=-\varepsilon_{0}+\frac{9}{2} B_{0} v_{0}\left[(1-x)^{2}+\left(B_{1}-1\right)(1-x)^{3}+\cdots\right] .
$$

Unless the analytic EOS form guarantees a minimum at $x$ $=1$, a fifth parameter is also needed to make the pressure vanish at $x=1$. Although it is very difficult to expand ( $x$ $>1$ ) a solid experimentally, both expansion and extreme compression can be achieved with theoretical calculations. Unless the equation of state contains a parameter $\varepsilon_{0}$ $=-\varepsilon(x=1)$, it cannot be fitted to bonding energy calculations. One commonly used solution to this problem is to add a constant to the UBER bonding energy of Eq. (8) (see, for example, Ref. 7), at the cost that the resulting $\varepsilon(x)$ no longer tends to zero as $x \rightarrow \infty$. Several four-parameter equations of state exist, as discussed in Ref. 8. But instead of cohesive energy they use $B_{2}$, the second derivative of the bulk modulus with respect to pressure at equilibrium, as a fourth parameter. These EOS's can probably provide a better description of the region very near $x=1$, but will not necessarily yield accurate cohesive energies. 
There are also problems specific to each EOS. The simple Murnaghan EOS provides considerable accuracy only when the range of data is limited to small compressions. The Birch EOS predicts positive pressures at the extreme compression limit only if $B_{1}>4$, which is not always the case. Holzapfel's H12 equation of state can have a negative exponential coefficient $\frac{3}{2}\left(B_{1}-1\right)-c_{0}$, which results in a physically unrealistic behavior of its $P(x)$ curve as $x \rightarrow \infty$ and makes $\varepsilon_{0}$ $=+\infty$. Yet another problem is that, while almost any reasonable equation of state can yield an accurate value of $B_{0}$ from a fit to theoretical or experimental data, it may take a very sophisticated one to yield an accurate $B_{1} \cdot{ }^{15,6} B_{2}$ is even harder to evaluate numerically than $B_{1}$.

One may also question the true "universality" of any equation of state. Even for simple metals in fixed crystal structures, the possibility of isostructural electronic phase transitions due to level crossings ${ }^{16,17}$ and electron topological transitions ${ }^{18}$ due to van Hove singularities shows that there can be no truly "universal" equation of state. Instead, we should perhaps introduce the idea of a "normal" equation of state, which predicts the high-pressure behavior of the material from its low-pressure behavior, in the absence of electronic transitions. The analytic equations of state mentioned above, and the stabilized jellium model to be described below, do not display electronic transitions. Once we have found a "normal" equation of state, we can use it to identify electronic transitions in real materials by looking for abnormal and abrupt deviations of the actual pressure from its analytic or "normal" EOS representation.

A realistic equation of state should predict a minimum negative pressure $P_{c}$ at a critical expansion $x_{c}$ where the uniform crystal would "break" under any further increase in applied tension. For example, the UBER predicts $x_{c}=1.22$ and $P_{c}=-0.23 B_{0}$ for $B_{1}=3$, while it predicts $x_{c}=1.11$ and $P_{c}=-0.11 B_{0}$ for $B_{1}=6$. Note that $B\left(x_{c}\right)=0$ and $B^{\prime}\left(x_{c}\right)$ $= \pm \infty$. When $x_{c}$ is sufficiently close to 1 , Taylor expansion yields $x_{c}=1+1 /\left(3 B_{1}\right)$. A liquid can support a uniform negative pressure ${ }^{19,20}$ in a metastable state.

The product $B v$ has been called the "physical hardness" of a solid. It correlates with resistance to scratching or puncture. $^{21,22}$ Pearson $^{22}$ has suggested that $B v$ might maximize at equilibrium (although the principle underlying his derivation has been questioned ${ }^{23}$ ). To investigate this possibility, we begin with the Taylor expansion (16) and find

$$
\begin{gathered}
P(x)=B_{0}\left[3(1-x)+\frac{3}{2}\left(3 B_{1}+1\right)(1-x)^{2}+\cdots\right], \\
B(x)=B_{0}\left[1+3 B_{1}(1-x)+\cdots\right], \\
B v=B_{0} v_{0}\left[1+3\left(B_{1}-1\right)(1-x)+\cdots\right] .
\end{gathered}
$$

Only for $B_{1}=1$ could $B v$ maximize at $x=1$ (as it does for a harmonic solid). For the exactly solved stabilized jellium model of the next section, $B_{1} \geqslant 3$. We note, however, that another correlate of physical hardness, $|\varepsilon(x)|$, does in fact maximize at $x=1$. The strongest correlate may be the shear modulus. ${ }^{24,25}$
$B_{1}$ is correlated with the Grüneisen anharmonicity constant

$$
\gamma=-\left.\frac{v}{\omega} \frac{d \omega}{d v}\right|_{v_{0}},
$$

where $\omega$ is an average phonon frequency. Dugdale and MacDonald ${ }^{26}$ found

$$
\gamma=\frac{1}{2}\left(B_{1}-1\right),
$$

an expression which properly vanishes for a harmonic crystal $\left(B_{1}=1\right)$. Here $\gamma$ in turn yields the contribution to the pressure from thermally excited phonons, $\gamma u / v$, where $u$ is the average energy per atom of the thermally excited phonons $\left(u \rightarrow 3 k_{B} T\right.$ as $\left.T \rightarrow \infty\right)$, according to the Mie-Grüneisen equation of state. ${ }^{27} \gamma$ is an important ingredient of the Hugoniot or shock curve, ${ }^{17,28} P(v, T)$ vs $v$, where the shocked temperature $T$ is determined by the shocked volume $v$ and by the initial volume and temperature. The thermal expansion coefficient is

$$
\alpha \equiv \frac{1}{v_{0}} \frac{d v_{0}}{d T}=\frac{\gamma c_{v}}{B_{0} v_{0}}
$$

where $c_{v}=(\partial u / \partial T)_{v}$.

In Appendix A we use the Grüneisen constant $\gamma$ to estimate the effect of phonon zero-point energy upon the equilibrium properties of a solid, finding 0\%-5\% effects for most of the simple metals and larger effects for Be.

\section{STABILIZED JELLIUM MODEL AND STABILIZED JELLIUM EQUATION OF STATE}

In this section, we will construct an equation of state motivated by the simplest realistic microscopic model for a solid: the stabilized jellium model of Ref. 2 and Appendix B. This model, which faithfully reproduces many trends in the properties of the simple metals, has been reviewed recently in Ref. 29. It provides a useful zero-order model for bulk ${ }^{2}$ and surface properties, ${ }^{30}$ cohesive and vacancy-formation energies, ${ }^{31,32}$ and size effects in clusters ${ }^{33,34}$ and thin films. ${ }^{35}$ Its relationship to other simple models such as the "ideal metal" ${ }^{36}$ has been discussed in Ref. 37. In Appendix B, we show that the original version ${ }^{38}$ of the "ideal metal" is the zero-valence $(z \rightarrow 0)$ limit of stabilized jellium. For the bulk simple metals, which are our main interest here, the stabilized jellium model has a long pedagogical history; cf. Ref. 39.

One of the simplest models for unstressed simple metals is the jellium model. ${ }^{40-42}$ In this model, each neutral atom is composed of $z$ valence electrons and an ion of nuclear charge $Z$ with $Z-z$ inert core electrons. Then the charge on the ions of the bulk solid is smeared into a uniform positive background, neutralized by valence electrons of density

$$
\bar{n}=\frac{3}{4 \pi r_{s}^{3}}
$$


TABLE II. Equilibrium properties for bulk stabilized jellium with valence $z=1$, found by analytic differentiation of the model energy (Ref. 2). Note that $B_{1}$ varies from 3 at $r_{s}=1.6$ to $10 / 3$ as $r_{s} \rightarrow \infty . B_{0}$ is approximately $9.18 \mathrm{Mbar} / r_{s}^{7 / 2}$ for $2<r_{s}<6$ [a formula which also works for real simple metals with $z \leqslant 4$ (Ref. 63)] and tends to $27.9 \mathrm{Mbar} / r_{s}^{4}$ as $r_{s} \rightarrow \infty$. The correlation energy $\varepsilon_{c}\left(r_{s}\right)$ is from Ref. 48. The model core radius $r_{c}$ is defined in Appendix B.

\begin{tabular}{ccccc}
\hline \hline $\begin{array}{c}r_{0}=r_{s} \\
\text { (bohr) }\end{array}$ & $\begin{array}{c}r_{c} \\
\text { (bohr) }\end{array}$ & $\begin{array}{c}\varepsilon_{0} \\
\text { (hartree) }\end{array}$ & $\begin{array}{c}B_{0} \\
(\text { Mbar })\end{array}$ & $B_{1}$ \\
\hline 2 & 0.51 & 0.399 & 0.8418 & 3.10 \\
3 & 1.16 & 0.292 & 0.2056 & 3.20 \\
4 & 1.76 & 0.230 & 0.0716 & 3.24 \\
5 & 2.35 & 0.189 & 0.0311 & 3.26 \\
6 & 2.94 & 0.161 & 0.0156 & 3.27 \\
\hline \hline
\end{tabular}

Here $r_{s}$ is the Seitz radius or density parameter. Although very simple and universal in the sense that all properties are controlled by a single parameter $r_{s}$, this model provides a realistic description of the cohesive and surface properties of metals only when $r_{s} \approx 4$ bohr (close to the density of sodium). At $r_{s}=4.2$, bulk jellium is stable (i.e., $P=0$ ). At sufficiently different densities, anomalies such as negative surface energy ${ }^{40}$ or negative bulk modulus ${ }^{43}$ arise. These problems are solved by introducing pseudopotential and Madelung corrections that stabilize the metal at its observed density. In the stabilized jellium model of Ref. 2, this pseudopotential is structureless. The difference between the pseudopotential of the ions in the lattice and the electrostatic potential of the uniform positive background, averaged over a Wigner-Seitz cell, is treated as a first-order perturbation to the total energy of jellium. The average energy per valence electron in the bulk is therefore

$$
\varepsilon=t_{s}+\varepsilon_{x c}+\bar{w}_{R}+\varepsilon_{M} .
$$

Here $t_{s}$ and $\varepsilon_{x c}$ are the kinetic and exchange-correlation energies per particle for a uniform electron gas. (Note that the model for the bulk solid is exactly solved, since the manyelectron effects in $\varepsilon_{x c}$ are transferred unchanged from jellium to stabilized jellium.) $\bar{w}_{R}$ is the average of the repulsive or non-Coulombic part of the electron-ion pseudopotential, which is chosen to make $\varepsilon$ minimize at the input $r_{s}$ for the input valence $z$. Here $\varepsilon_{M}$ is the average Madelung or electrostatic energy of a collection of point ions embedded in a uniform negative background. $t_{s}$ and $\varepsilon_{x c}$ depend only upon $r_{s}$, while $\bar{w}_{R}$ and $\varepsilon_{M}$ also depend upon the valence $z$. The simplicity of the jellium model is partly lost as the bulk properties now depend upon $z$ as well as $r_{s}$, although the surface properties still depend only upon $r_{s}$. Table II shows $\varepsilon_{0}, B_{0}$, and $B_{1}$ as functions of $r_{s}$ for stabilized jellium with $z=1$. The choice $z=1$ yields realistic values for $B_{0}$ in all the simple metals, even the polyvalent ones.

Each of the terms of Eq. (22) depends upon volume per electron as a simple power:

$$
\bar{w}_{R} \sim v^{-1}, \quad t_{s} \sim v^{-2 / 3}, \quad \varepsilon_{M} \sim v^{-1 / 3}, \quad \varepsilon_{x} \sim v^{-1 / 3},
$$

and the correlation energy $\varepsilon_{c}=\varepsilon_{x c}-\varepsilon_{x}$ varies roughly as $v^{0}$. The stabilized jellium equation of state (SJEOS) is thus

$$
\text { SJEOS: } \quad \varepsilon(x)=\frac{a}{x^{3}}+\frac{b}{x^{2}}+\frac{c}{x}+d,
$$

making

$$
\text { SJEOS: } \quad P(x)=\frac{1}{3 v_{0}}\left[\frac{3 a}{x^{6}}+\frac{2 b}{x^{5}}+\frac{c}{x^{4}}\right] \text {. }
$$

The energy parameters $a, b, c$, and $d$ are related to the equilibrium parameters $B_{0}, B_{1}, v_{0}$, and cohesive energy $\varepsilon_{0}$ via the equilibrium conditions

$$
\begin{gathered}
\varepsilon(x=1)=a+b+c+d=-\varepsilon_{0}, \\
P(x=1)=\frac{3 a+2 b+c}{3 v_{0}}=0, \\
B(x=1)=\frac{18 a+10 b+4 c}{9 v_{0}}=B_{0}, \\
B^{\prime}(x=1)=\frac{108 a+50 b+16 c}{27 B_{0} v_{0}}=B_{0}^{\prime}=B_{1} .
\end{gathered}
$$

These conditions provide a system of four linear equations for $a, b, c$, and $d$. The SJEOS values for these parameters are found by fitting the EOS to experimental or theoretical bonding energies in a narrow range near equilibrium. Then one can find the equilibrium parameters from Eqs. (26)-(29). Or, vice versa, using given equilibrium parameters (experimental or found with some EOS) and the equations

$$
\begin{gathered}
a=\frac{9}{2} B_{0} v_{0}\left(B_{1}-3\right), \\
b=\frac{9}{2} B_{0} v_{0}\left(10-3 B_{1}\right), \\
c=-\frac{9}{2} B_{0} v_{0}\left(11-3 B_{1}\right), \\
d=-\varepsilon_{0}+\frac{9}{2} B_{0} v_{0}\left(4-B_{1}\right),
\end{gathered}
$$

one can reconstruct the SJEOS curve in order to study the high-compression behavior of the material. For stabilized jellium with $z=1$ (although not necessarily for real solids), we find $a>0, b>0, c<0$, and $d \approx 0$ on the scale of $\varepsilon_{0}$.

Table II shows the equilibrium properties of stabilized jellium with valence $z=1$. Because Eqs. (24) and (25) reflect the physics of stabilized jellium, they provide nearly perfect fits to the energy and pressure (Fig. 1) of this model system, unlike the standard equations of state (5)-(10). For true stability at $x=1$, Eq. (24) requires $a>0$ and thus $B_{1}>3$, consistent with the observation that real solids seldom if ever have $B_{1}<3 .{ }^{6}$ The H02 of Eq. (9) also requires $B_{1}>3$, while the UBER of Eq. (7) only requires $B_{1}>1$. 


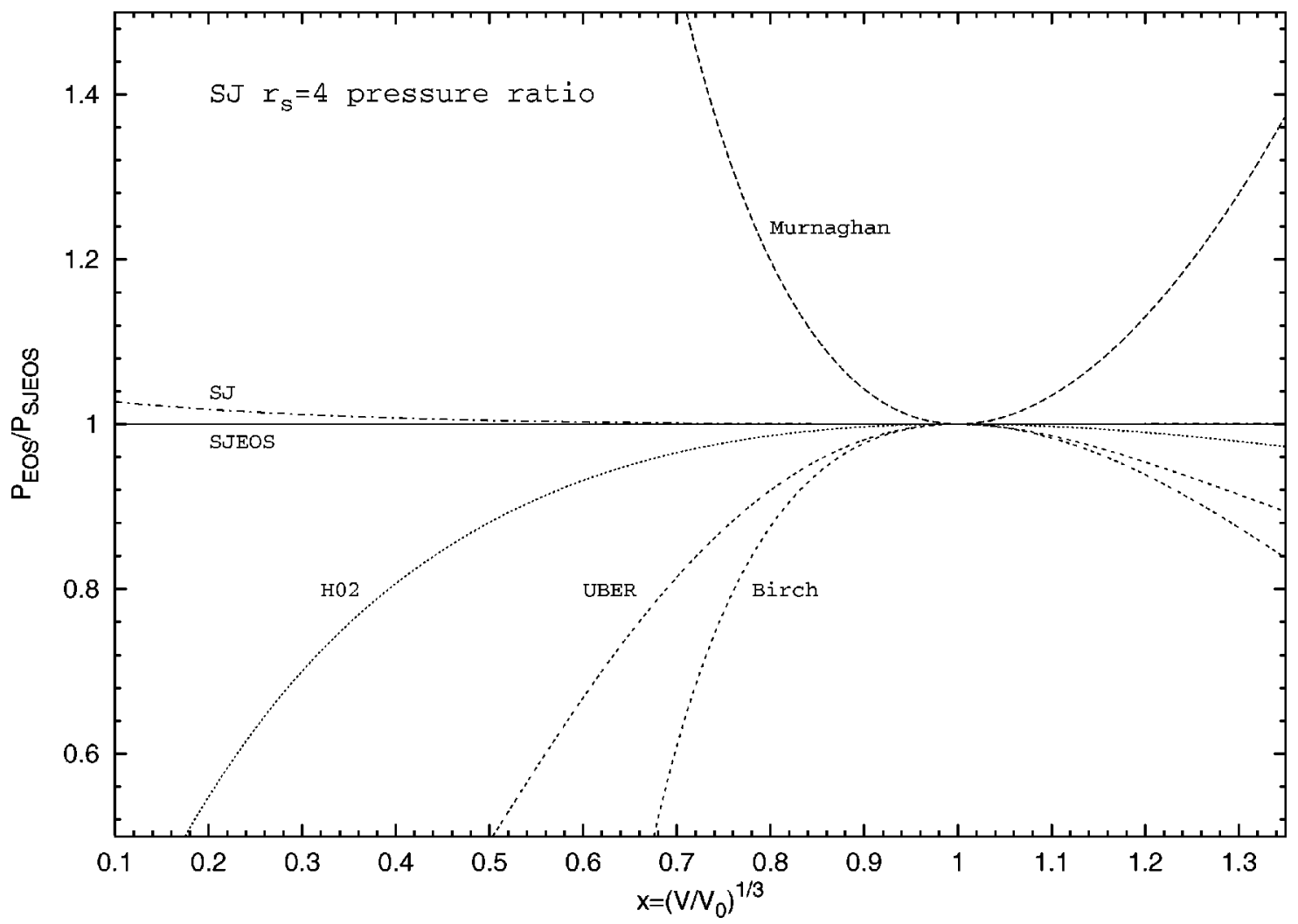

FIG. 1. Pressure ratio $P_{E O S} / P_{S J E O S}$ vs compression ratio $x$ for stabilized jellium with valence $z=1$ and equilibrium density parameter $r_{s}=4$, using the equilibrium parameters $B_{0}$ and $B_{1}$ from Table II. Note that 15 Mbar $>P>0$ for $0.43<x<1$.

For ordinary jellium, we must replace Eq. (22) by $\varepsilon=t_{s}$ $+\varepsilon_{x c}$. Our SJEOS of Eqs. (24) and (25) also describes ordinary jellium, with $r_{s}=4.18$ bohrs, $\varepsilon_{0}=0.078$ hartree, $B_{0}$ $=0.0147$ Mbar, and $B_{1}=3.0$.

An EOS in the form of Eq. (24) was used earlier by Teter and co-workers ${ }^{44}$ to fit their first-principles total-energy pseudopotential calculations of cohesive energies of several silica structures. They found it to fit the data better than the Murnaghan and Birch equations of state. However, they did not relate their choice of EOS to the stabilized jellium or any other microscopic model.

The stabilized jellium equation of state is physically plausible for simple metals with $0.6 \leqq x \leqq 1.1$. In this range, it is reasonable to treat the electron-ion pseudopotential as a weak perturbation. Then the leading small- $x$ contribution to the energy is the pseudopotential repulsion term $\bar{w}_{R}$ or $a / x^{3}$, and the leading small- $x$ contribution to the pressure is $a /\left(v_{0} x^{6}\right)$. In the stabilized jellium model with $z=1, \bar{w}_{R}$ and $a$ are positive (and thus $B_{1} \geqslant 3$ ) for all metals with equilibrium $r_{s} \geqslant 1.6$. At $r_{s}=1.6$, the density parameter of monatomic metallic hydrogen (see Fig. 1 of Ref. 2), $\bar{w}_{R}$ and $a$ vanish and $B_{1}=3$.

For small $x(\ll 1)$, the ion cores overlap and the pseudopotential picture fails. When the overlap is very strong, the core electrons pressure ionize into the valence band, ${ }^{45}$ and the effective valence increases from $z$ toward $Z$. The true $x \rightarrow 0$ pressure is presumably the $P_{F G} / x^{5}$ Fermi gas pressure included in the H12 EOS, as in standard treatments of white dwarf stars. In this limit, $B \rightarrow \frac{5}{3} P$ and $B^{\prime} \rightarrow \frac{5}{3}$. However, this limit is only approached under non-laboratory conditions.

For large $x(>1.2)$, the electron-ion pseudopotential in a real metal is not a weak perturbation. It binds $z$ valence electrons closely around each ion, and as a result $\varepsilon(x)$ and $P(x)$ for a real metal approach zero as $x \rightarrow \infty$ much more rapidly than for stabilized jellium [Eqs. (24) and (25)]. In the stabilized jellium model, the positive background is always uniform, even under extreme expansion. Thus $\varepsilon_{0}$ for stabilized jellium corresponds in real metals not to the cohesive energy but to the bulk binding energy of valence electrons and ions. A more realistic but less simple model for expansion would split the background into spheres or polyhedra representing individual atoms, as in the current version of the ideal-metal model. ${ }^{36}$

\section{AUGMENTED STABILIZED JELLIUM EQUATION OF STATE FOR REAL SOLIDS}

Here we shall modify the SJEOS of Eq. (24) to account for the differences between real simple metals and stabilized jellium, as explained at the end of the previous section.

For $x \leqslant 1$, we would retain Eq. (24) for pseudopotential energies. For all-electron energies, we simulate the effect of ion-core overlap by introducing a function $g(x)$,

ASJEOS: $\quad \varepsilon(x)=\frac{a}{x^{3}} g(x)+\frac{b}{x^{2}}+\frac{c}{x}+d \quad(x \leqslant 1)$. 
To recover the SJEOS as $x \rightarrow 1$, the function $g(x)$ must approach 1 in this limit, with $g^{\prime}(1)=g^{\prime \prime}(1)=g^{\prime \prime \prime}(1)=0$. But as $x \rightarrow 0$, the function $g(x)$ must approach $f x$ (where $f$ is a positive constant) so that the $a / x^{3}$ term in $\varepsilon$ gets replaced by $f a / x^{2}$, reflecting the disappearance of the core repulsion and the appearance of an extra free-electron kinetic energy when the outer core electrons are gradually liberated under intense pressure. (For metallic hydrogen, which has no core, $a=0$ and the ASJEOS correctly reduces to the SJEOS.)

A function that satisfies these expectations is

$$
g(x)=1+\alpha(1-x)^{4}-\beta(1-x)^{5}+\gamma(1-x)^{6},
$$

where

$$
\begin{gathered}
\alpha=5 f-\frac{1}{2} h-15, \\
\beta=9 f-h-24, \\
\gamma=4 f-\frac{1}{2} h-10 .
\end{gathered}
$$

So far, $f$ and $h$ are arbitrary constants. We might want to choose $f$ to recover the Fermi-gas limit presented after Eq. (10). For $\mathrm{Li}$, the simple metal with the least-bound core, we would then find $f=6.8$. But for the other simple metals at achievable pressures, the Fermi-gas limit (in which all the core electrons are liberated) is so far away as to be irrelevant.

A previous study of local pseudopotentials for the simple metals ${ }^{46}$ found that the repulsive contribution to the effective electron-ion interaction decayed as $e^{-r / R}$, where $R$ is the decay length of the highest-energy core orbital of $s$ or $p$ symmetry, and that $r_{0} \approx 7 R$ where $r_{0}=z^{1 / 3} r_{s}$ is the radius of the atomic cell in the unstressed solid $\left[v_{0}=(4 \pi / 3) r_{0}^{3}\right]$. Writing

$$
\frac{r}{R}=\frac{r_{0}}{R} \frac{r}{r_{0}},
$$

we estimate $f \approx 7$. Then, for exponentially overlapped ion cores, we would expect $g(x) \approx 1-e^{-f x}$ where $f \approx 7$. We mimic this behavior by using Eqs. (35)-(38) with $f=6.8$ and $h=42$, making $\alpha=-2.0, \beta=-4.8$, and $\gamma=-3.8$.

The ASJEOS pressure for $x \leqslant 1$ is

ASJEOS: $\quad P(x)=\frac{1}{3 v_{0}}\left[\frac{3 a}{x^{6}} g(x)+\frac{2 b-a g^{\prime}(x)}{x^{5}}+\frac{c}{x^{4}}\right]$ $(x \leqslant 1)$.

Then Eqs. (30) and (31) imply that

$$
\lim _{x \rightarrow 0} P(x)=\frac{2(f a+b)}{3 v_{0} x^{5}}=\frac{3 B_{0}\left(3.8 B_{1}-10.4\right)}{x^{5}},
$$

which is properly positive for all $B_{1}>2.74$.

For $x>1$, we allow for an exponential decay of $\varepsilon(x)$ as $x \rightarrow \infty$ :

$$
\text { ASJEOS: } \begin{aligned}
\varepsilon(x)= & {\left[\frac{A}{x^{3}}+\frac{B}{x^{2}}+\frac{C}{x}+D+(A+B+C+D)\right.} \\
& \times F(x-1)] e^{-F(x-1)} \quad(x>1),
\end{aligned}
$$

where we choose $F$ as discussed in the next paragraph. As $x \rightarrow \infty$, our $\varepsilon(x)$ tends to zero exponentially from below, much like the UBER. At $x=1$, we match $\varepsilon(x)$ and its first three derivatives from $x<1$ and $x>1$. Then we have a linear-fitting problem for the coefficients $a, b, c, d$, and a nonlinear-fitting problem for $v_{0}$. Detailed ASJEOS expressions for $A, B, C, D$ and the pressure for $x>1$ are presented in Appendix C.

The decay length for the density of the free atom is roughly $L_{a}=1 /\left[2 \sqrt{-2 m \varepsilon_{H O}^{L D A} / \hbar^{2}}\right]$, where $\varepsilon_{H O}^{L D A}$ is the highest-occupied orbital energy calculated with the local density approximation for exchange and correlation. Using $\varepsilon_{H O}^{L D A}$ values from Ref. 47 , we have evaluated $F=r_{0} / L_{a}$ for all the elements in Table I.

Fourth- and higher-order derivatives of the ASJEOS $\varepsilon(x)$ are discontinuous at $x=1$. We can still estimate the true derivatives there by averaging the derivatives (Appendix A) for $x=1-\delta$ and $x=1+\delta$, where $\delta$ is an infinitesimal. If the only data available are for $x \leqslant 1$ (e.g., measured pressures under compression), we can simply fit to Eq. (34) or Eq. (39).

\section{FITTING THE ASJEOS TO ALL-ELECTRON CALCULATIONS FOR THE BONDING ENERGIES OF Al, Li, AND Mo}

As we mentioned above, one way to use an EOS is to fit it to a given set of energy vs compression (or pressure vs compression) data points around equilibrium, to find the equilibrium properties of the material such as $B_{0}$ and $B_{1}$. Here we use this method to test our SJEOS and ASJEOS on different metals. We also investigate the performance of earlier equations of state, in comparison with the SJEOS and ASJEOS.

The simple fitting algorithm we use is initially to select a small (arbitrary) number of consecutive data points at values of $x$ around (or near) equilibrium $(x=1)$ and make a linear fit to these data points. The fitting criterion is the root mean square (rms) error of the fit. If the rms error is larger (smaller) than the estimated numerical precision of the data points fitted, the fitting range in $x$ is narrowed (extended), and the whole fitting procedure is repeated again, until the rms error is comparable with the numerical precision.

Fitting over a range of $x$ wider than that defined at the end of the previous paragraph would improve the global fit, including the high-compression regime, but worsen the estimates of $B_{0}$ and $B_{1}$. Here $B_{1}$ is particularly sensitive to the fitting range.

We first tested our SJEOS and ASJEOS on Al and Li. These simple metals are not so unlike stabilized jellium.

A series of all-electron, full-potential local density approximation $^{48}$ (LDA) electronic structure calculations 
TABLE III. Equilibrium properties from the fits to LCGTO total energies calculated within the local density approximation. Note that $B_{1}$ depends sensitively upon the EOS used to make the fit. The fitting ranges are given in the captions to Figs. 2, 4, and 6.

\begin{tabular}{cclll}
\hline \hline & & $\mathrm{Al}$ & $\mathrm{Li}$ & Mo \\
\hline$\varepsilon_{0}$ & SJEOS & 0.1542 & 0.0750 & 0.4597 \\
(hartree) & ASJEOS & 0.1542 & 0.0750 & 0.4597 \\
& Expt. & 0.125 & 0.060 & 0.251 \\
& & & & \\
$v_{0}$ & SJEOS & 107.9 & 127.9 & 101.8 \\
$\left(\right.$ bohr $\left.^{3}\right)$ & ASJEOS & 107.9 & 127.9 & 101.8 \\
& Expt. & 112.0 & 142.5 & 105.5 \\
& & & & \\
$B_{0}$ & SJEOS & 0.8205 & 0.1509 & 2.924 \\
$\left(\mathrm{Mbar}^{2}\right)$ & ASJEOS & 0.8242 & 0.1505 & 2.941 \\
& Expt. & 0.794 & 0.133 & 2.73 \\
& & & & \\
$B_{1}$ & SJEOS & 4.74 & 3.34 & 4.01 \\
& ASJEOS & 4.96 & 3.44 & 4.20 \\
& Expt. & 4.7 & 3.5 & 4.7 \\
\hline \hline
\end{tabular}

were carried out for fcc $\mathrm{Li}$ and fcc Al, using the linear combination of Gaussian-type orbitals-fitting function (LCGTOFF) technique, ${ }^{49-53}$ as implemented in the program GTOFF. ${ }^{54}$ (The LCGTO-FF method is distinguished from other LCGTO techniques by its use of independent auxiliary GTO basis sets to fit the charge density and the exchangecorrelation integral kernels.) The LCGTO-FF method is particularly well suited for calculating the cold EOS over a wide pressure range because, unlike some other electronic structure techniques used for crystals, the LCGTO-FF method does not require an a priori partitioning of the electron states between "core', (nonhybridizing) and "band" (hybridizing) states. Rather, all electron states are allowed to hybridize fully, ensuring a continuous representation of core states as they are forced into the continuum under pressure.

The precision of any LCGTO-FF calculation will, of course, be largely determined by the selection of the three GTO basis sets. In this work, relatively rich uncontracted orbital basis sets were used for $\mathrm{Li}(9 s 4 p 2 d)$ and $\mathrm{Al}$ $(11 s 7 p 2 d)$. A single $9 s$ GTO basis set was used to fit both the charge density and the exchange-correlation kernels for $\mathrm{Li}$, while an $11 \mathrm{~s}$ fit basis was used for Al. For the more compressed or expanded volumes, the various basis sets were scaled to avoid linear dependency while maintaining adequate flexibility in the diffuse regions. (These basis sets can be obtained from JCB.) All requisite Brillouin zone integrations were carried out on a uniform mesh with 256 irreducible $k$ points in the fcc Brillouin zone, using a Gaussian-broadened histogram integration technique, with a broadening factor of $5 \mathrm{mH}$. The self-consistency cycle was iterated until the total energy varied by less than $0.002 \mathrm{mH} /$ atom.

The lattice constants at which we calculated LCGTO energies and pressures were chosen before we had either a new EOS or a fitting procedure. For $\mathrm{Li}$, total energies were first calculated for 12 lattice constants ranging from 6.5 bohrs to 8.5 bohrs. For higher compressions, total energies were calculated at pairs of lattice constants lying sufficiently close to allow an accurate determination of the pressure from finite differencing. Eight such pairs of lattice constants were considered ranging from 6.5 bohrs to 3.0 bohrs, and pressures were calculated using each pair. Finally, total energies were calculated for three expanded lattice constants: 9.0, 9.5, and 10.0 bohrs.

A similar approach was taken for Al. Energies were cal-

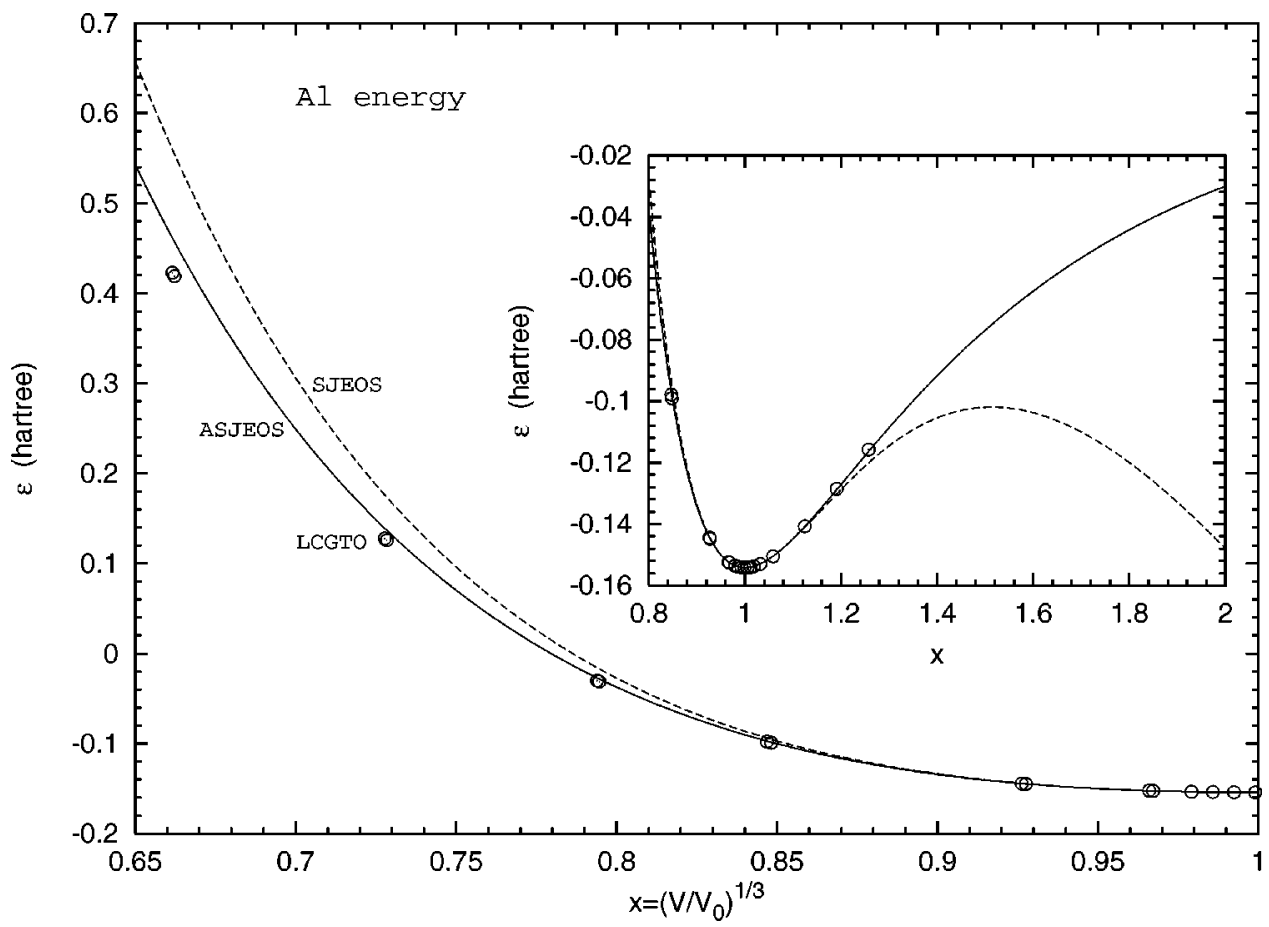

FIG. 2. Bonding energy $\varepsilon$ vs $x$ for Al. The circles are the LCGTO data within the local density approximation, the dashed line is the SJEOS, and the solid line is the ASJEOS. We have used as inputs $F$ from Table I and $\varepsilon_{0}, v_{0}, B_{0}$, and $B_{1}$ (Table III) from the ASJEOS fit to 12 LCGTO data points in the range $0.93 \leqslant x \leqslant 1.06$. 


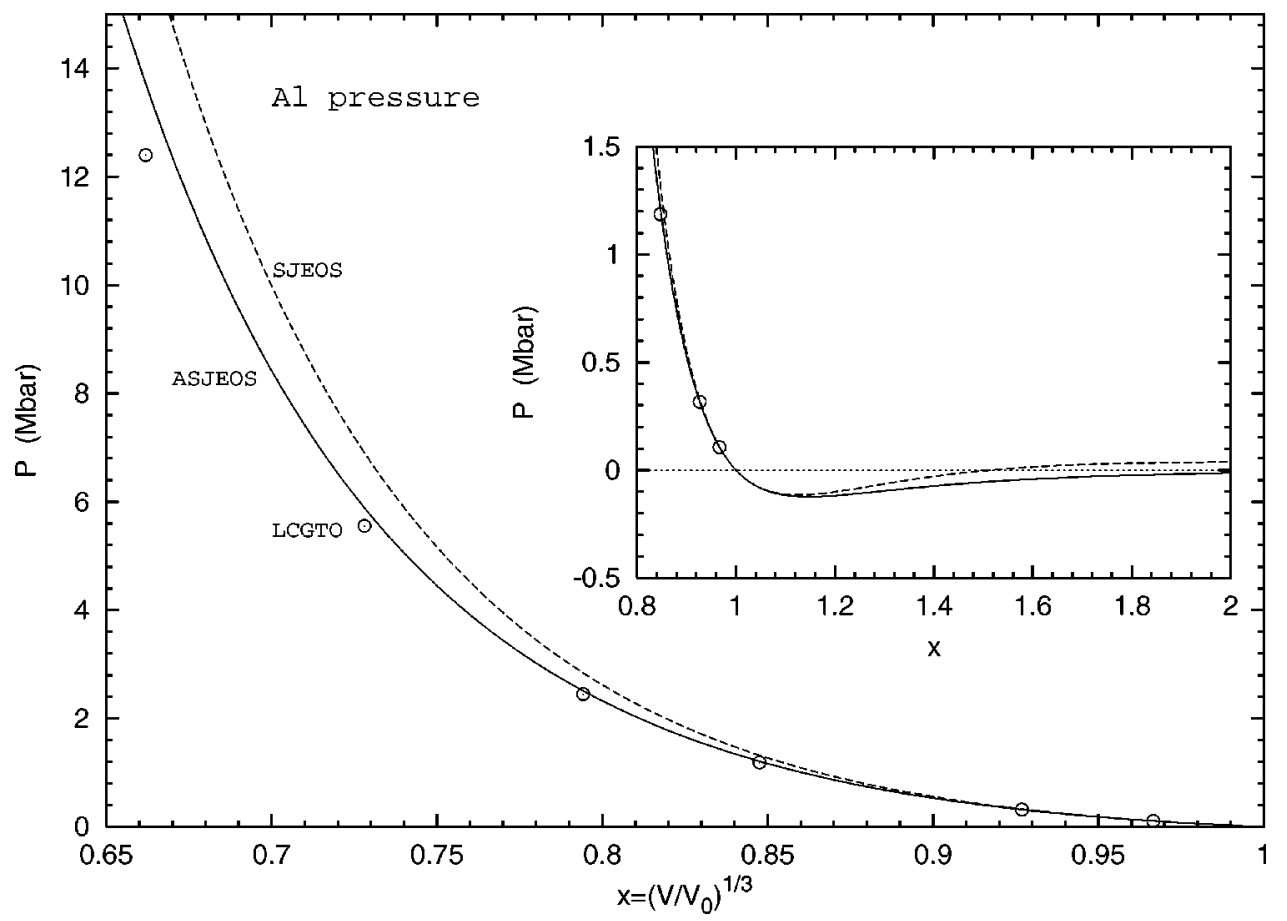

FIG. 3. Pressure $P$ vs $x$ for Al. See caption of Fig. 2. The LCGTO pressures were found by numerical differentiation of the LCGTO energies.

culated for 12 compressed lattice constants ranging from 6.0 bohrs to 7.8 bohrs, ten pairs of high-compression lattice constants ranging from 7.3 bohrs to 3.0 bohrs (also used to calculate pressure), and four expanded lattice constants 8.0, 8.5, 9.0, and 9.5 bohrs.

Although our SJEOS and ASJEOS are constructed for the simple metals, they may apply more broadly to transition metals like Mo or even to nonmetals. For bcc Mo energies, the scalar-relativistic LCGTO-FF calculations of Ref. 55 were used, with lattice constants ranging from 4.374 bohrs to 6.10 bohrs. In addition, the pressure was calculated for the highest compression point $(x=0.74)$ using numerical differentiation.

The estimated numerical precision of the LCGTO data for $\mathrm{Al}$ and $\mathrm{Li}$ is $5 \times 10^{-6}$ hartrees, and by construction this is also the ASJEOS fit error in the narrow fitting range around $x=1$. The fitting range for each solid is reported in the caption of Figs. 2, 4, and 6. The LCGTO data is used to test various equations of state in Table III and Figs. 2-8. Since the ASJEOS provides a good fit over the whole range of $x$ considered, we have used the ASJEOS to estimate $B_{0}$ and $B_{1}$ for use in all the tested equations of state. The figures show

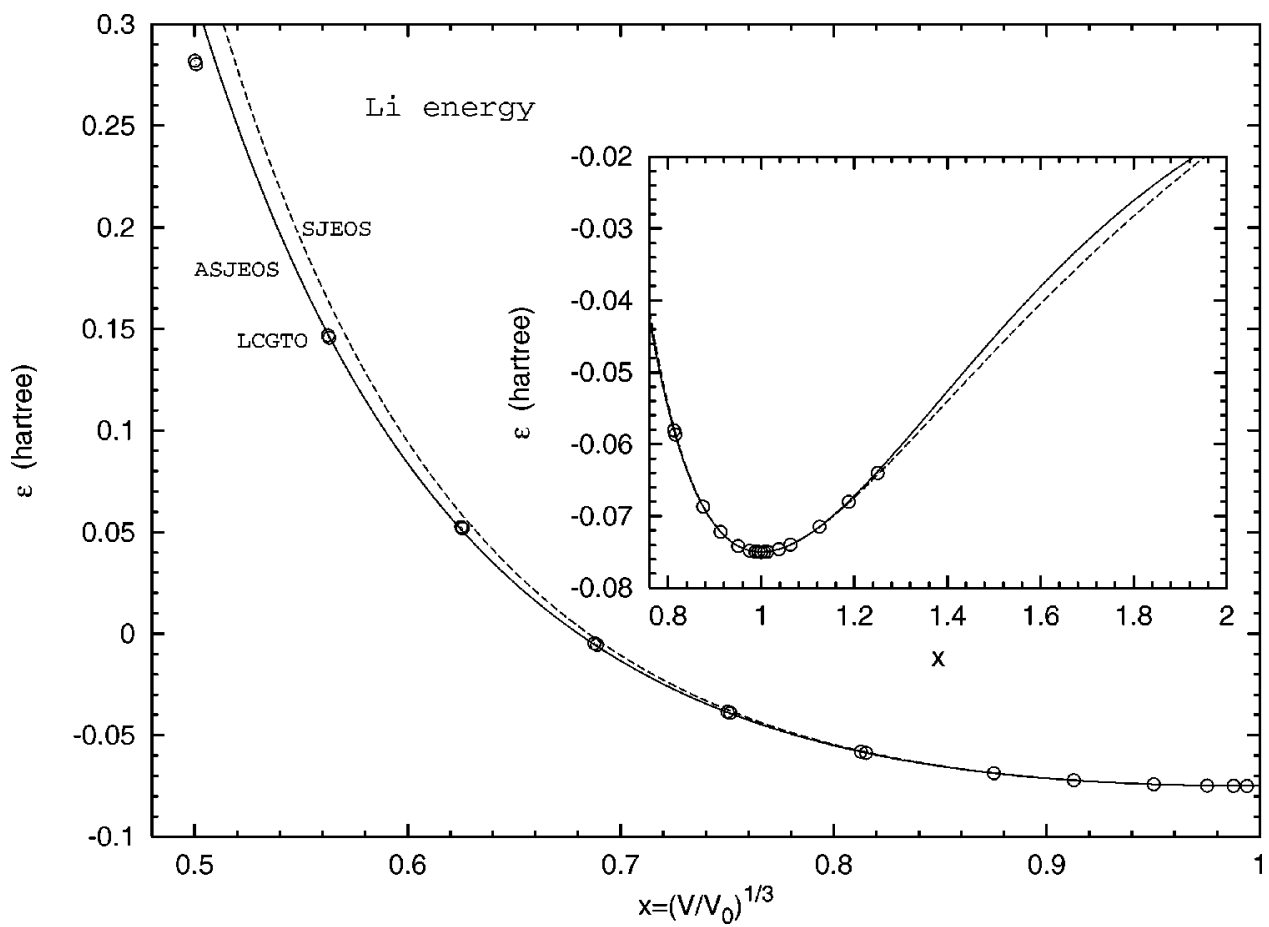

FIG. 4. Bonding energy $\varepsilon$ vs $x$ for Li. We have used as inputs $F$ from Table I and $\varepsilon_{0}, v_{0}, B_{0}$, and $B_{1}$ (Table III) from the ASJEOS fit to 12 LCGTO data points in the range $0.88 \leqslant x \leqslant 1.13$. 


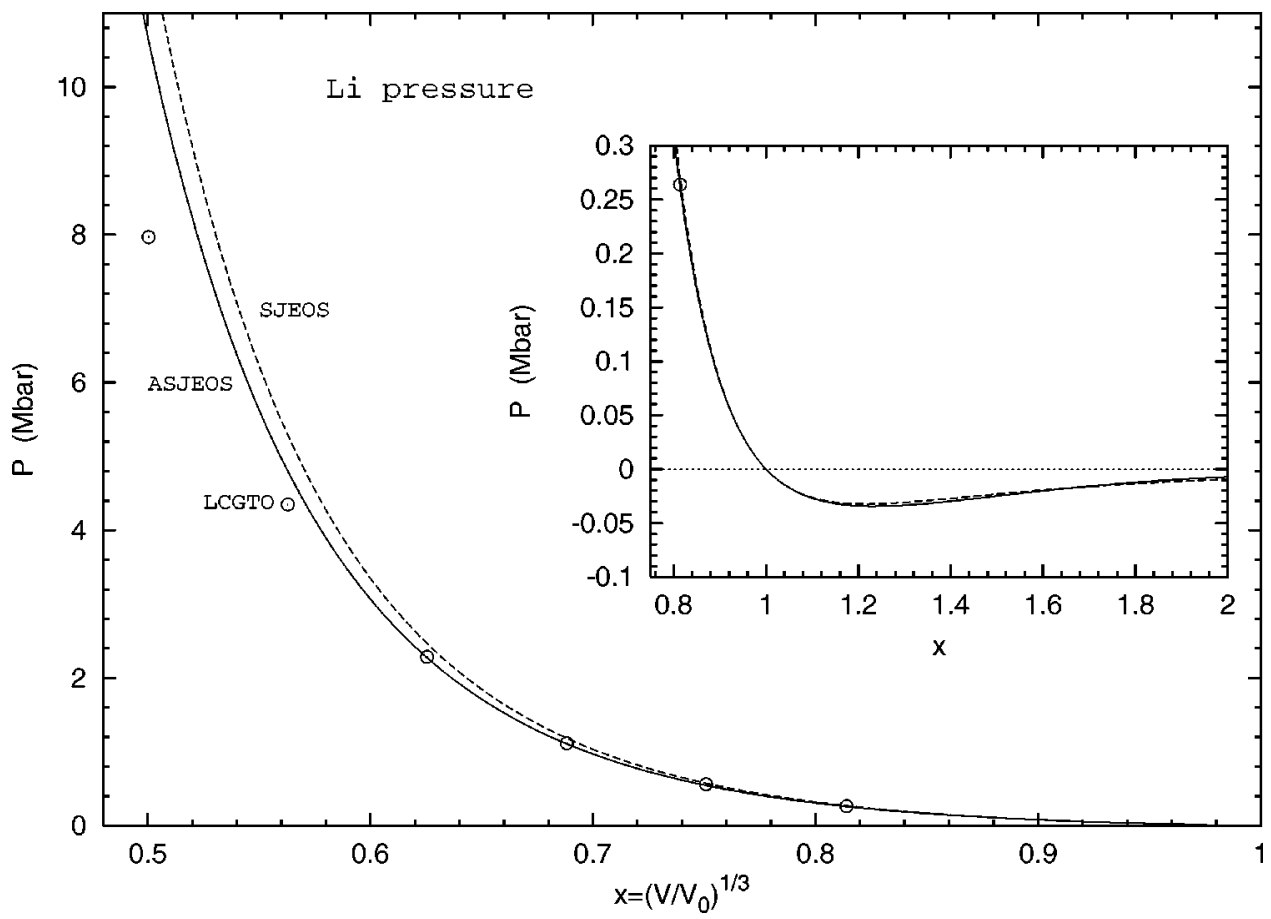

FIG. 5. Pressure $P$ vs $x$ for Li. See caption of Fig. 4. The LCGTO pressures were found by numerical differentiation of the LCGTO energies.

that the ASJEOS matches the LCGTO data better than the SJEOS and better than the earlier equations of state.

Finally, we point out that our LCGTO cohesive energies $\varepsilon_{0}$ have been calculated by subtracting the energy per atom of the solid in equilibrium from the energy of the free atom, both calculated in the spin-unpolarized local density approximation (LDA). The more usual procedure, which finds the energy of the free atom in the local spin density (LSD) approximation, yields a more realistic cohesive energy but introduces a spurious kink into the binding energy curve at the value of $x>1$ where the system begins to spin polarize. Nei- ther our new EOS nor the earlier ones could possibly describe that spurious kink.

\section{CORRECTING ERRORS IN $V_{0}, B_{0}, B_{1}$ AND THE EOS THAT ARISE FROM DENSITY FUNCTIONAL APPROXIMATIONS}

Modern electronic structure calculations require a density functional approximation (DFA) for the exchangecorrelation energy, e.g., local density ${ }^{48}$ or generalized gradient $^{56}$ approximations. Near equilibrium, the results of

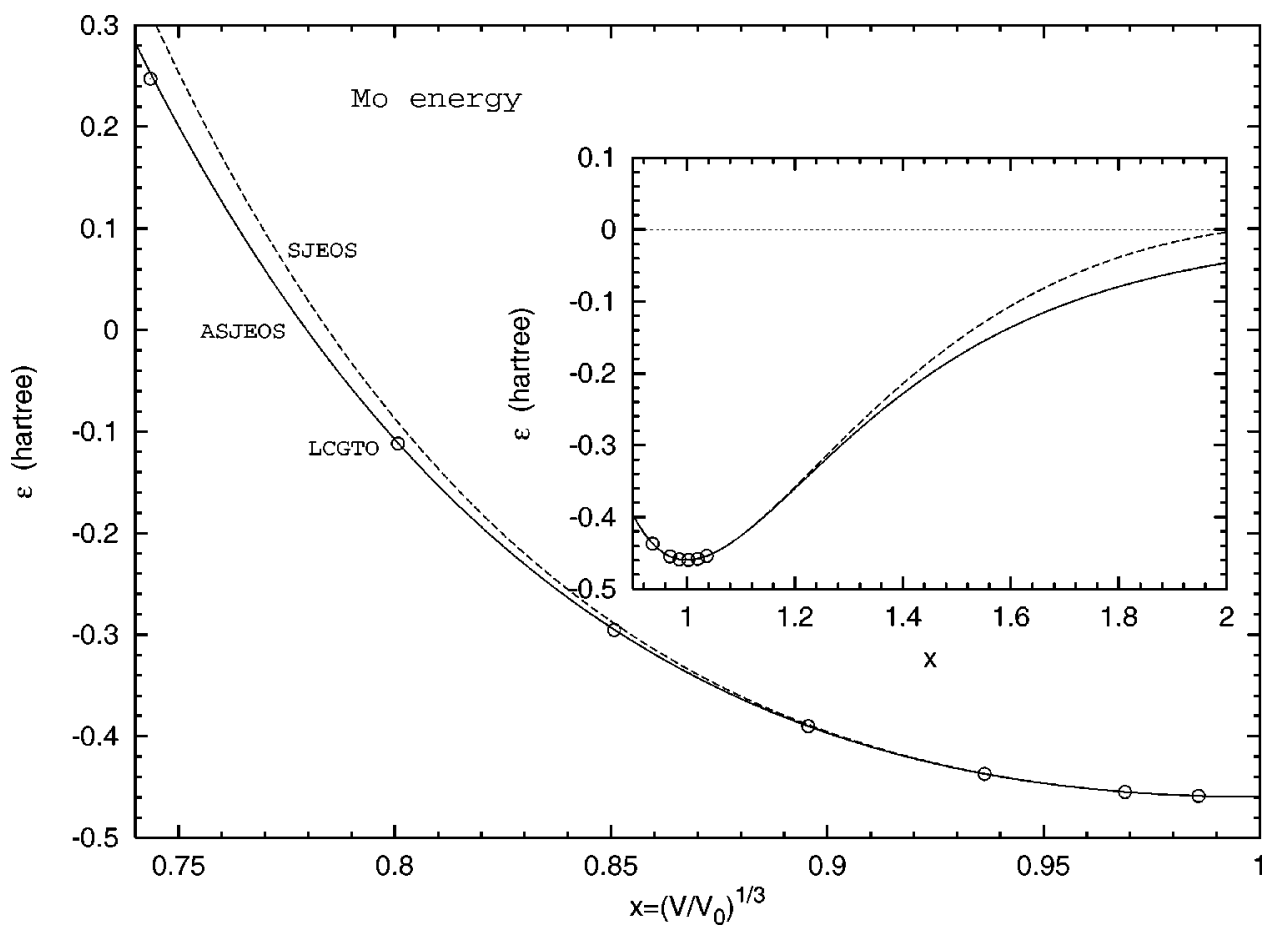

FIG. 6. Bonding energy $\varepsilon$ vs $x$ for Mo. We have used as inputs $F$ from Table I and $\varepsilon_{0}, v_{0}, B_{0}$, and $B_{1}$ (Table III) from the ASJEOS fit to seven LCGTO data points in the range $0.90 \leqslant x \leqslant 1.04$. At the highest-compression LCGTO point shown, the pressure is 14.4 Mbar according to both ASJEOS and numerical differentiation of the LCGTO data. 


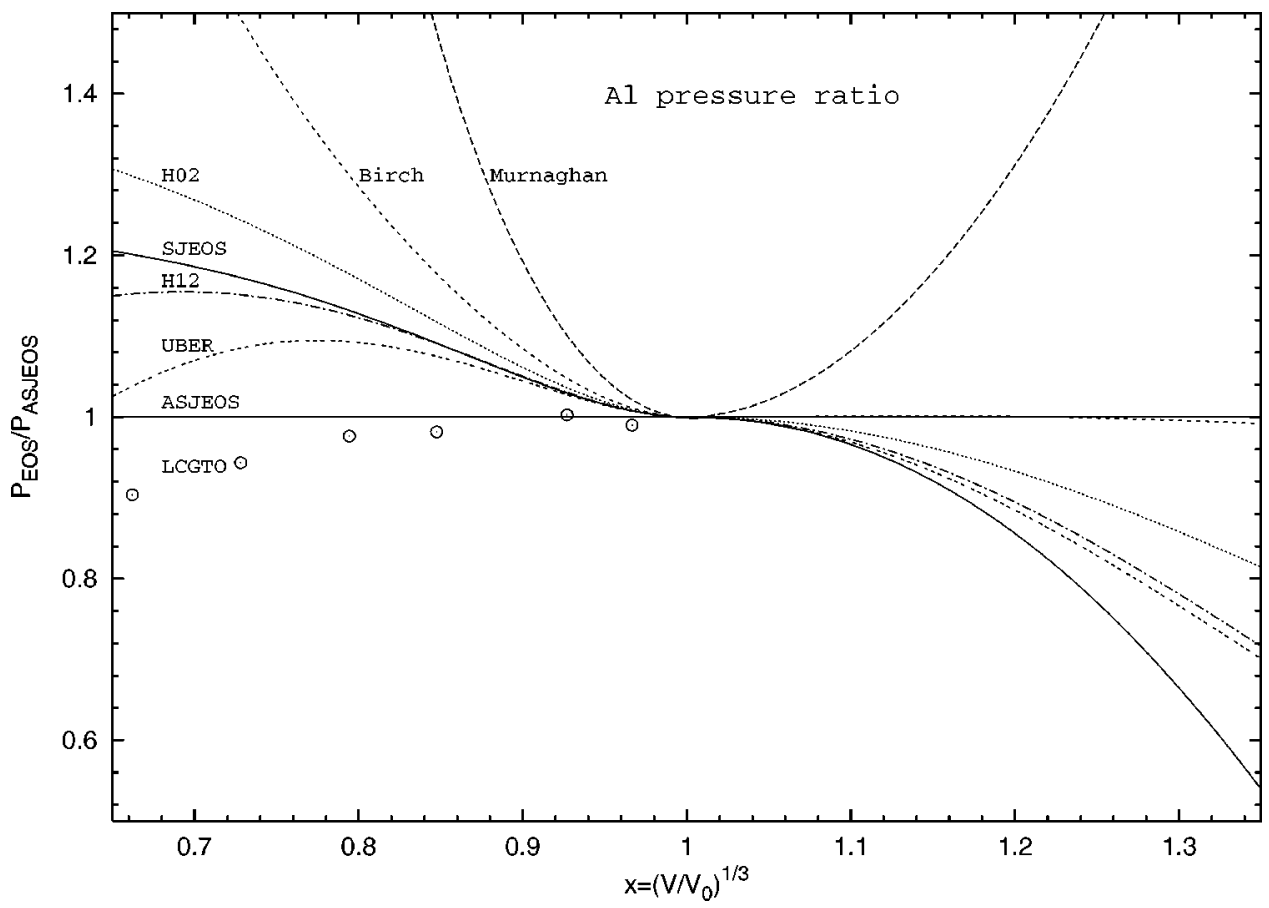

FIG. 7. Pressure ratio $P_{E O S} /$ $P_{\text {ASJEOS }}$ vs $x$ for $\mathrm{Al}$, using the equilibrium parameters from the caption of Fig. 2.

such a calculation may be fitted to the SJEOS of Eq. (25):

$$
P^{D F A}(x)=\frac{1}{3 v_{0}^{D F A}}\left[\frac{3 a^{D F A}}{x^{6}}+\frac{2 b^{D F A}}{x^{5}}+\frac{c^{D F A}}{x^{4}}\right],
$$

where in this section

$$
x \equiv\left(\frac{v}{v_{0}^{D F A}}\right)^{1 / 3} .
$$

Then the bulk modulus $B_{0}^{D F A}$ and its pressure derivative $B_{1}^{D F A}$ may be found from Eqs. (28) and (29).

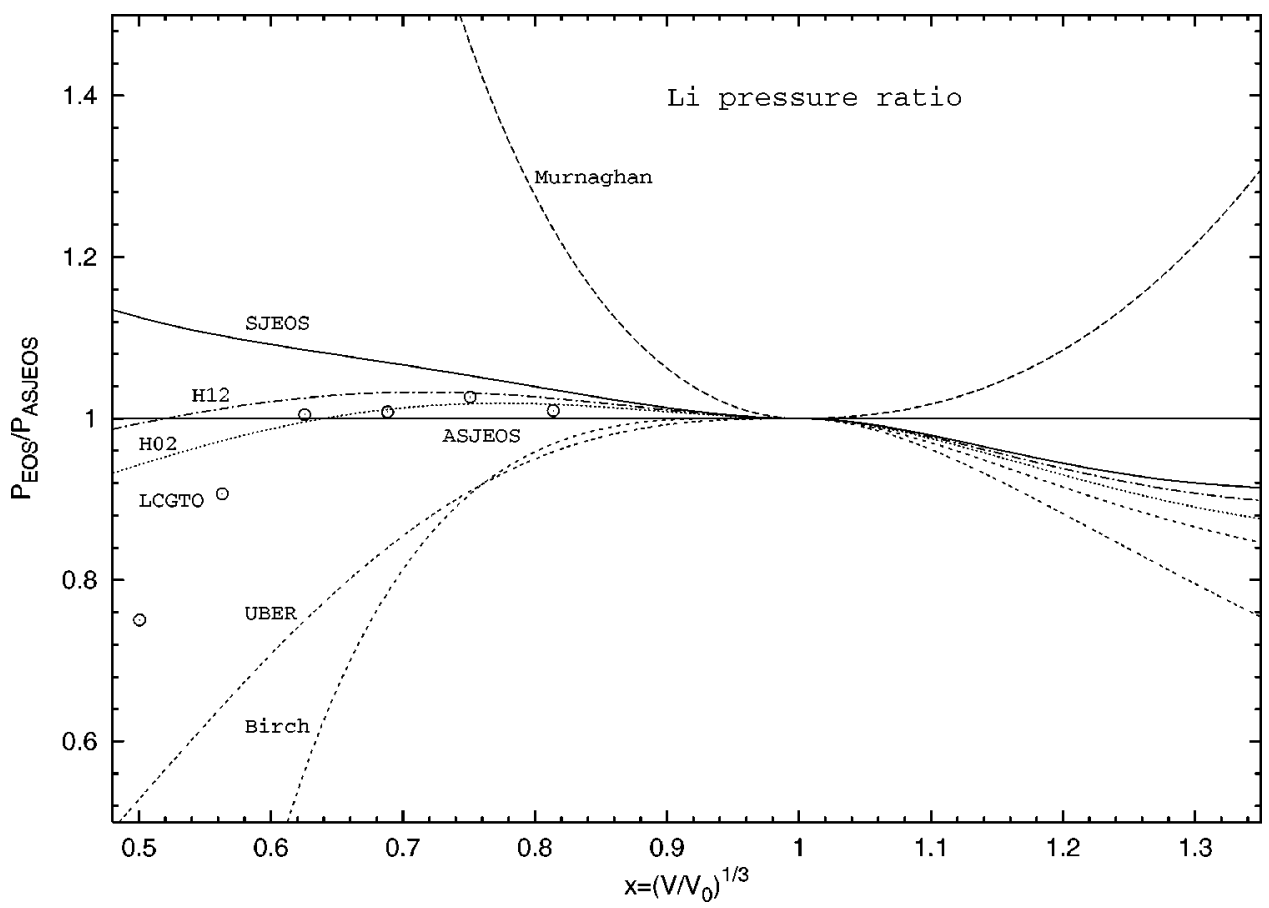

In comparison with the experimental equilibrium volume $v_{0}^{\text {expt }}$, the theoretical volume $v_{0}^{D F A}$ can be in error by a typical $4 \%$ or $5 \% .{ }^{57}$ The main source of this error, identified by Fuchs et $a l .{ }^{58}$ as the core-valence interaction (see also Ref. 59 and Fig. 9 of Ref. 60), is in pseudopotential theory embedded mainly in the $a^{D F A}$ term of Eq. (42), i.e., in the pseudopotential acting on the valence pseudo-orbitals. Thus, if we know $v_{0}^{\text {expt }}$, we can write a corrected EOS

$$
\widetilde{P}(x)=\frac{1}{3 v_{0}^{D F A}}\left[\frac{3 \tilde{a}}{x^{6}}+\frac{2 b^{D F A}}{x^{5}}+\frac{c^{D F A}}{x^{4}}\right],
$$

FIG. 8. Pressure ratio $P_{E O S} /$ $P_{\text {ASJEOS }}$ vs $x$ for $\mathrm{Li}$, using the equilibrium parameters from the caption of Fig. 4. The LCGTO values display an electronic phase transition (Refs. 64 and 65) at $x$ $\approx 0.6$. 
with $\tilde{a}$ adjusted to make $\widetilde{P}(x)$ vanish when $v=v_{0}^{\text {expt }}$ or $x$ $=x_{0}$ :

$$
\begin{gathered}
3 \tilde{a}=-2 b^{D F A} x_{0}-c^{D F A} x_{0}^{2}, \\
x_{0}=\left(\frac{v_{0}^{\text {expt }}}{v_{0}^{D F A}}\right)^{1 / 3} .
\end{gathered}
$$

[We could also replace $a^{D F A}$ by $\tilde{a}$ and $\varepsilon_{0}^{D F A}$ by $\varepsilon_{0}^{\text {expt }}$ in the ASJEOS of Eq. (39).] From Eqs. (3), (4), and (44), we find the corrected bulk modulus $\widetilde{B}_{0}$ and its pressure derivative $\widetilde{B}_{1}$ at $v=v_{0}^{\text {expt }}$ :

$$
\begin{gathered}
\widetilde{B}_{0}=-\frac{2}{9 v_{0}^{D F A}}\left[\frac{b^{D F A}}{x_{0}^{5}}+\frac{c^{D F A}}{x_{0}^{4}}\right], \\
\widetilde{B}_{1}=\frac{\frac{11}{3} b^{D F A}+\frac{10}{3} c^{D F A} x_{0}}{b^{D F A}+c^{D F A} x_{0}} .
\end{gathered}
$$

A different way to make this correction was proposed recently by van de Walle and Ceder (WC). ${ }^{61}$ We discuss this work as an example of a phenomenological approach to this correction. Unlike our Eq. (44), which changes the shape of the pressure, they simply shifted this curve up or down by a constant $\Delta$ :

$$
\widetilde{P}^{W C}(x)=P^{D F A}(x)+\Delta,
$$

where $\Delta$ is chosen to make $\widetilde{P}^{W C}\left(x_{0}\right)=0$. Equation (49) has a history; for example, it was used by Boettger and Trickey ${ }^{62}$ to bring the local density pressures for solid $\mathrm{Ne}$ into agreement with experiment. With the help of Eq. (42), we find

$$
\begin{aligned}
\widetilde{B}_{0}^{W C} & =-\frac{2}{9 v_{0}^{D F A}}\left[\frac{b^{D F A}}{x_{0}^{5}}\left(\frac{6}{x_{0}}-5\right)+\frac{c^{D F A}}{x_{0}^{4}}\left(\frac{3}{x_{0}^{2}}-2\right)\right] \\
& =B^{D F A}\left(x_{0}\right)
\end{aligned}
$$

Equation (50), which amounts to evaluating $B^{D F A}(x)$ at the experimental equilibrium point $x_{0}$, has also been widely used.

Comparing Eqs. (44) and (49), we see that only our physically motivated equation (44) satisfies the exact condition $\widetilde{P}(x=\infty)=0$. For $\mathrm{Li}, \mathrm{Al}$, and Mo, our equation (47) is more accurate than Eq. (50) (Table IV).

We favor our equations (47) and (48) for the correction, not only because they work, but also because they are based upon microscopic insight into the origin of the density functional error. The inputs $b^{D F A}$ and $c^{D F A}$ can be found from Eqs. (31) and (32), if $B_{0}^{D F A}$ and $B_{1}^{D F A}$ are known. Neglecting the difference between $x_{0}^{5}$ and $x_{0}^{4}$ in Eq. (47) leads to the simpler estimate

$$
\widetilde{B}_{0} \approx \frac{B_{0}^{D F A}}{x_{0}^{4}}
$$

\begin{tabular}{|c|c|c|c|c|c|c|}
\hline Metal & $v_{0}^{D F A}$ & $\begin{array}{l}v_{0}^{\text {expt } t} \\
\left.\mathrm{r}^{3}\right)\end{array}$ & $B_{0}^{D F A}$ & $\begin{array}{r}\widetilde{B}_{0}^{W C} \\
(\mathrm{M}\end{array}$ & $\begin{array}{l}\widetilde{B}_{0} \\
\text { ar) }\end{array}$ & $B_{0}^{\text {expt }}$ \\
\hline $\mathrm{Al}$ & 108.0 & 112.0 & 0.820 & 0.686 & 0.741 & 0.794 \\
\hline $\mathrm{Li}$ & 128.0 & 142.5 & 0.150 & 0.103 & 0.130 & 0.133 \\
\hline Mo & 101.8 & 105.5 & 2.94 & 2.52 & 2.72 & 2.73 \\
\hline
\end{tabular}

TABLE IV. Correcting the bulk modulus from the local density functional approximation (DFA) via Eqs. (47) and (50), which employ the experimental equilibrium volume $v_{0}^{\text {expt }}$ and the ASJEOS DFA equilibrium parameters from Table III. A more recent experimental value for $\mathrm{Al}$ is $B_{0}^{\text {expt }}=0.727 \mathrm{Mbar}$ (Ref. 76).

which can also be derived phenomenologically by replacing $\varepsilon^{D F A}(x)$ by $x_{0}^{-3} \varepsilon^{D F A}\left(x-x_{0}+1\right)$, i.e., by shifting the DFA energy curve rigidly along the $x$ axis to bring its minimum to the experimental equilibrium value of $x$ and then rescaling it by $x_{0}^{-3}$.

\section{DISCUSSION AND CONCLUSIONS}

In a sense, the construction of a universal or normal equation of state is only an exercise in curve fitting. Even so, it should reflect our understanding of the physics of condensed matter under compression and expansion. Here we have used the simple physics of the simple metals as a guide for this construction.

Like the Murnaghan, Birch, UBER, and H02 equations of state, but perhaps more reliably, our SJEOS of Eq. (25) and our ASJEOS of Eq. (39) predict the pressure $P(x)$ of a compressed solid in terms of only two material parameters $B_{0}$ and $B_{1}$. This remarkable economy of description is shared by certain microscopic models for the simple metals: not only the stabilized jellium model of Ref. 2, but also the universal local pseudopotential model of Ref. 63. In the latter model, $B_{0}$ determines $r_{s}$ via Fig. 2 of Ref. 63, then $r_{s}$ and $B_{1}$ determine $z$ via Fig. 3 of Ref. 63, and finally $r_{s}$ and $z$ determine the structured local pseudopotential and everything that can be calculated from it.

For the UBER of Eq. (7),

$$
\text { UBER: } \quad \frac{x^{2} P(x)}{3(1-x)}=B_{0} H\left(\left(B_{1}-1\right)(1-x)\right) \text {, }
$$

where $H(y)=1+\frac{3}{2} y+O\left(y^{2}\right)$. But for our SJEOS of Eq. (25) and for our ASJEOS of Eq. (39),

$$
\frac{x^{2} P(x)}{3(1-x)}=B_{0}\left[H_{1}(1-x)+\left(B_{1}-1\right) H_{2}(1-x)\right],
$$

where $H_{1}(y)=1+O\left(y^{2}\right)$ and $H_{2}(y)=\frac{3}{2} y+O\left(y^{2}\right)$. Thus we have two universal functions $\left(H_{1}\right.$ and $\left.H_{2}\right)$ instead of one $(H)$ and a linear fitting problem instead of a nonlinear one. The linearity is an advantage, since the solution of linear algebraic equations is simple and unique. Although only $B_{0}$ and $B_{1}$ enter our ASJEOS pressure for $x<1$, the cohesive energy $\varepsilon_{0}$ has an effect upon our values for $B_{0}$ and $B_{1}$ through our fitting procedure around $x=1$. Thus, the cohesive energy is an indirect input to the ASJEOS for a compressed solid. 
For the stabilized jellium model (Fig. 1), the SJEOS is clearly more accurate than the other equations of state. For the bonding energies of the real metals and for their pressures under expansion, the ASJEOS (which inputs the cohesive energy $\varepsilon_{0}$ ) is best. For the pressure of compressed $\mathrm{Al}$ (Fig. 7), the ASJEOS seems best but the UBER is also very good. For the pressure of compressed Li (Fig. 8), the ASJEOS seems best but $\mathrm{H} 02$ and $\mathrm{H} 12$ are also very good for $x \geqslant 0.6$; for $x \leqslant 0.6$, these equations of state appear to fail because of a $2 s \rightarrow 2 p$ electronic transition. ${ }^{64-66}$ For Mo, the ASJEOS is almost perfect. We see no reason to continue to use the Murnaghan and Birch equations, since even the simpler SJEOS is much more realistic.

The physically motivated SJEOS and ASJEOS also provide a promising way [Eqs. (44)-(48)] to correct EOS errors that arise from the use of approximate density functionals.

Once we have fitted energies or pressures to a good analytic EOS form, we can use the residuals of the it $^{9}$ or the pressure ratios (Figs. 7 and 8) to identify subtle electronic phase transitions. Figure 8 shows a sudden pressure softening in Li for $x \leqslant 0.6$ due to the $s \rightarrow p$ transition. ${ }^{64-66}$ Figure 7 suggests a more gradual pressure softening in $\mathrm{Al}$ for $x \leqslant 0.8$ due to the lowering and filling of the $d$ bands ${ }^{67}$ which in turn may cause structural phase transitions. ${ }^{7,67}$

Although we have taken $f$ and $h$ of Eqs. (36)-(38) to be fixed universal parameters, it is also possible to treat $a \cdot f$ and $a \cdot h$ as linear-fit parameters for each solid. While $\mathrm{Al}, \mathrm{Li}$, and Mo do not seem to need this extra flexibility, some other materials might require it. Those least likely to require this extra flexibility are the simple metals in close-packed or nearly close-packed crystal structures, our paradigm materials.

FORTRAN subroutines for the SJEOS and ASJEOS are available on request from perdew@tulane.edu.

Finally, we note that there are significant discrepancies between our results for compressed $\mathrm{Al}$ and those of Hama and Suito. ${ }^{8}$ For $x<0.8$, they plot their results on a logarithmic scale which conceals differences, but at $x=0.8$ they find that the UBER pressure is only about $2 \%$ higher than their calculated pressure in the local density approximation, while we find it about $10 \%$ higher (Fig. 7). Their values for $v_{0}, B_{0}$, and $B_{1}$ were fitted as ours were to a narrow range of calculations around $x=1$ (see the caption of Fig. 2), but differ significantly from ours. For example, they find $B_{0}=0.726$ Mbar while we find $B_{0}=0.824 \mathrm{Mbar}$, in better agreement with the linearized-augmented-plane-wave value $(0.840$ Mbar) found in Ref. 57.

\section{ACKNOWLEDGMENTS}

We thank R.G. Pearson and M. Ross for correspondence. The work of J.P.P. and A.B.A. was supported by the National Science Foundation under Grant No. DMR 98-10621. The work of J.C.B. and R.C.A. was supported by the U.S. Department of Energy under Contract No. W-7405-ENG-36. C.F. acknowledges the Portuguese Praxis program (Project No. Praxis/2/2.1/Fis/473/94) and the hospitality of Tulane University during a sabbatical leave in 1997/1998.

\section{APPENDIX A: EFFECT OF PHONON ZERO-POINT ENERGY ON THE EQUILIBRIUM PROPERTIES OF A SOLID AND ON THE EOS}

The phonon zero-point energy per atom is $\frac{3}{2} \hbar \omega$, where $\omega$ is an average phonon frequency: $\hbar \omega=\frac{3}{4} k_{B} \Theta_{D} \cdot{ }^{27}$ Here $\Theta_{D}$ is the Debye temperature. Using a superscript zero to indicate a quantity in the absence of phonon zero-point energy, we find

$$
\begin{gathered}
\varepsilon(v)=\varepsilon^{0}(v)+\frac{3}{2} \hbar \omega(v), \\
P(v)=P^{0}(v)+\frac{3}{2} \gamma(v) \frac{\hbar \omega(v)}{v}, \\
B(v)=B^{0}(v)+\frac{3}{2}\left[\gamma^{2}(v)+\gamma(v)-v \frac{d \gamma(v)}{d v}\right] \frac{\hbar \omega(v)}{v},
\end{gathered}
$$

where

$$
\gamma(v)=-\frac{v}{\omega} \frac{d \omega}{d v}
$$

is the Grüneisen parameter discussed at the end of Sec. II.

We immediately find

$$
\frac{\Delta \varepsilon_{0}}{\varepsilon_{0}}=-\frac{9}{8} \frac{k_{B} \Theta_{D}}{\varepsilon_{0}}
$$

for the fractional change in $\varepsilon_{0}$ due to zero-point vibration. Taylor expansion around $v_{0}$ and use of Eq. (A4) gives

$$
\frac{\Delta v_{0}}{v_{0}}=\frac{9}{16}\left(B_{1}-1\right) \frac{k_{B} \Theta_{D}}{B_{0} v_{0}}
$$

To evaluate $d \gamma / d v$, we need Eq. (19) of Ref. 26:

$$
\gamma(v)=-1+\frac{1}{2} \frac{B\left(1+B^{\prime}\right)-\frac{10}{9} P}{B-\frac{2}{3} P}
$$

(so that $\gamma \rightarrow \frac{2}{3}$ as $x \rightarrow 0$ ). We find

$$
\left.v \frac{d \gamma}{d v}\right|_{v_{0}}=\frac{2}{9}-\frac{1}{3} B_{1}-\left.\frac{B_{0}}{2} \frac{d B^{\prime}}{d P}\right|_{v_{0}} .
$$

Equation (5.5) of Ref. 6 gives the UBER value

$$
B_{0} B_{2}=\left.B_{0} \frac{d B^{\prime}}{d P}\right|_{v_{0}}=\frac{19}{36}-\frac{1}{2} B_{1}-\frac{1}{4} B_{1}^{2},
$$

but we shall use instead the arithmetic average of the ASJEOS values

$$
\begin{array}{r}
B_{0} B_{2}=\left.B_{0} \frac{d B^{\prime}}{d P}\right|_{v_{0}}=-\frac{74}{9}+5 B_{1}-B_{1}^{2}+\frac{4}{3} \alpha\left(B_{1}-3\right) \\
\left(x \rightarrow 0^{-}\right),(\mathrm{A} 10)
\end{array}
$$


TABLE V. Effect of phonon zero-point energy upon the equilibrium properties of the simple metals, using experimental values of $v_{0}, \varepsilon_{0}, B_{0}$, and $B_{1}$ from Table I, and experimental Debye temperatures $\Theta_{D}$ from Ref.74.

\begin{tabular}{lcccc}
\hline \hline Metal & $\Theta_{D}(\mathrm{~K})$ & $\Delta \varepsilon_{0} / \varepsilon_{0}$ & $\Delta v_{0} / v_{0}$ & $\Delta B_{0} / B_{0}$ \\
\hline $\mathrm{Cs}$ & 38 & -0.005 & 0.003 & -0.009 \\
$\mathrm{Rb}$ & 56 & -0.006 & 0.005 & -0.014 \\
$\mathrm{Tl}$ & 79 & -0.004 & 0.003 & -0.012 \\
$\mathrm{~K}$ & 91 & -0.009 & 0.008 & -0.021 \\
$\mathrm{~Pb}$ & 105 & -0.005 & 0.002 & -0.011 \\
$\mathrm{In}$ & 108 & -0.004 & 0.003 & -0.011 \\
$\mathrm{Ba}$ & 110 & -0.006 & 0.003 & -0.008 \\
$\mathrm{Sr}$ & 147 & -0.008 & 0.004 & -0.013 \\
$\mathrm{Na}$ & 158 & -0.014 & 0.013 & -0.031 \\
$\mathrm{Sn}$ & 200 & -0.006 & 0.005 & -0.025 \\
$\mathrm{Ca}$ & 230 & -0.012 & 0.006 & -0.018 \\
$\mathrm{Ga}$ & 320 & -0.011 & 0.007 & -0.021 \\
$\mathrm{Li}$ & 344 & -0.020 & 0.024 & -0.045 \\
$\mathrm{Mg}$ & 400 & -0.026 & 0.011 & -0.036 \\
$\mathrm{Al}$ & 428 & -0.012 & 0.009 & -0.033 \\
$\mathrm{Be}$ & 1440 & -0.042 & 0.043 & -0.140 \\
$\mathrm{Mo}$ & 450 & -0.006 & 0.003 & -0.011 \\
\hline \hline
\end{tabular}

$$
\begin{aligned}
B_{0} B_{2}=\left.B_{0} \frac{d B^{\prime}}{d P}\right|_{v_{0}}= & -\frac{74}{9}+5 B_{1}-B_{1}^{2}-\frac{1}{3}\left(2 F^{2}+16 F\right. \\
& \left.-4 F B_{1}\right)+\varepsilon_{0} \frac{F^{2}(6+F)^{2}}{81 B_{0} v_{0}} \quad\left(x \rightarrow 0^{+}\right),
\end{aligned}
$$

where $\alpha=-2.0$. [Incidentally, $\alpha=0$ in Eq. (A10) gives the SJEOS value, and $\alpha=-\frac{3}{2}$ makes Eq. (A10) agree with Eq. (A9) for the harmonic crystal, for which $B_{1}=1$ and Eq. (A9) is exact.] Using Eq. (A8), we find

$$
\frac{\Delta B_{0}}{B_{0}}=-\left[\frac{1}{2}\left(B_{1}-1\right)+\frac{2}{B_{1}-1}\left(\frac{2}{9}-\frac{1}{3} B_{1}-\frac{1}{2} B_{0} B_{2}\right)\right] \frac{\Delta v_{0}}{v_{0}} .
$$

Table $\mathrm{V}$ shows our estimates for the simple metals. Our $\Delta B_{0} / B_{0}$ for $\mathrm{Mg}$ is close to the value $(-0.028)$ calculated in Ref. 68. If we had neglected the contribution from $v d \gamma / d v$, as in Ref. 69, we would have found $\Delta B_{0} / B_{0}=-0.015$ for $\mathrm{Mg}$.

The rather large effect we predict for Be improves the agreement between theoretical (LDA and GGA) (Ref. 70) and experimental values for the equilibrium volume and bulk modulus, and might explain the anomalous surface lattice relaxation $^{70,71}$ of this metal.

Away from equilibrium, we can use Eq. (A2) to estimate the contribution of phonon zero-point vibration to the pressure. Integration of Eq. (A4) gives

$$
\omega(v)=\omega\left(v_{0}\right) e^{q(v)}
$$

where

$$
q(v)=\int_{v}^{v_{0}} d v \frac{\gamma(v)}{v}
$$

For a simple estimate, note that, for $0 \leqslant v \leqslant v_{0}$,

$$
\frac{2}{3} \leqslant \gamma(v) \leqslant \frac{1}{2}\left(B_{1}-1\right)
$$

Thus the extra pressure in Eq. (A2) is bounded between two limits,

$$
\frac{\frac{3}{4} k_{B} \Theta_{D}\left(v_{0}\right)}{v_{0} x^{5}} \leqslant \frac{3}{2} \gamma(v) \frac{\hbar \omega(v)}{v} \leqslant \frac{3}{4}\left(B_{1}-1\right) \frac{\frac{3}{4} k_{B} \Theta_{D}\left(v_{0}\right)}{v_{0} x^{3\left(B_{1}+1\right) / 2}},
$$

where the upper bound should be close for $v \approx v_{0}$ and the lower one for $v \rightarrow 0$. For $\mathrm{Al}$ and $\mathrm{Li}$ at the volumes studied in this paper, the extra pressure of Eq. (A16) is negligible.

\section{APPENDIX B: STABILIZED JELLIUM AND THE IDEAL METAL}

In the stabilized jellium model of Ref. 2, the total energy as a functional of the electron density $n(\mathbf{r})$ is

$$
\begin{aligned}
E_{S J}\left[n, n_{+}\right]= & E_{J}\left[n, n_{+}\right]+\left(\varepsilon_{M}+\bar{w}_{R}\right) \int d^{3} r n_{+}(\mathbf{r}) \\
& +\langle\delta v\rangle_{W S} \int d^{3} r \Theta(\mathbf{r})\left[n(\mathbf{r})-n_{+}(\mathbf{r})\right]
\end{aligned}
$$

where $\varepsilon_{M}=-9 e^{2} z^{2 / 3} /\left(10 r_{s}\right), \bar{w}_{R}=2 \pi e^{2} \bar{n} r_{c}^{2}$ (in which $r_{c}$ is a constant determined by $z$ and by the equilibrium value of $\bar{n}$ or $\left.r_{s}\right)$, and $n_{+}(\mathbf{r})=\bar{n} \Theta(\mathbf{r})$ is the positive background density. $\Theta(\mathbf{r})$ equals 1 inside a zero-thickness surface and zero outside. $E_{J}\left[n, n_{+}\right]$is the energy functional for ordinary jellium, and $\langle\delta v\rangle_{W S}$ is a pseudopotential correction averaged over a Wigner-Seitz cell:

$$
\langle\delta v\rangle_{W S}=\tilde{\varepsilon}+\varepsilon_{M}+\bar{w}_{R},
$$

where $\tilde{\varepsilon}=3 e^{2} z^{2 / 3} /\left(5 r_{s}\right)$.

In the zero-valence limit $(z \rightarrow 0)$, we have $\varepsilon_{M} \rightarrow 0, \tilde{\varepsilon}$ $\rightarrow 0, \bar{w}_{R} \rightarrow\langle\delta v\rangle_{W S}$, and the energy functional becomes

$$
\begin{aligned}
E_{S J}\left[n, n_{+}\right] \rightarrow & E_{J}\left[n, n_{+}\right]+\langle\delta v\rangle_{W S} \int d^{3} r n_{+}(\mathbf{r}) \\
& +\langle\delta v\rangle_{W S} \int d^{3} r \Theta(\mathbf{r})\left[n(\mathbf{r})-n_{+}(\mathbf{r})\right]
\end{aligned}
$$

But $\Theta(\mathbf{r}) n_{+}(\mathbf{r})=n_{+}(\mathbf{r})$; therefore,

$$
\lim _{z \rightarrow 0} E_{S J}\left[n, n_{+}\right]=E_{J}\left[n, n_{+}\right]+\langle\delta v\rangle_{W S} \int d^{3} r \Theta(\mathbf{r}) n(\mathbf{r}),
$$


which is the original ideal-metal energy functional of Ref. 38. After it was realized that the original ideal metal is unstable $36,72,73$ in the bulk, the model was modified to its current metastable form. ${ }^{36,73}$ The stabilized jellium model with $z=1$ is truly stable ${ }^{72}$ for $r_{s}>1.6$.

Our conclusion that the original ideal metal is the zerovalence limit of the stabilized jellium model is consistent with Soler's 72 way of forming the ideal metal by " grinding," the ions into a fine powder spread uniformly over the crystal: as $z \rightarrow 0$, the radius $z^{1 / 3} r_{s}$ of the atomic cell also vanishes.

\section{APPENDIX C: DETAILED ASJEOS EXPRESSIONS}

When fit to theoretical data, the ASJEOS of Eqs. (34) and (41) has only $a, b$, and $d$ as independent fitting parameters. The parameter $c$ is replaced by

$$
c=-3 a-2 b
$$

through the equilibrium condition (27).

Furthermore, equating the zeroth, first, second, and third derivatives of both sides of the ASJEOS [Eqs. (34) and (41)] at $x=1$ and solving the resulting system of four linear equations for the parameters $A, B, C$, and $D$, we find

$$
\begin{aligned}
A= & \frac{1}{6}\left[2 a\left(F^{3}+6 F^{2}-9 F+3\right)+b\left(F^{3}+6 F^{2}-6 F\right)\right. \\
& \left.-d\left(F^{3}+6 F^{2}\right)\right],
\end{aligned}
$$

$$
\begin{aligned}
B= & -\frac{1}{2}\left[2 a\left(F^{3}+7 F^{2}-9 F\right)+b\left(F^{3}+7 F^{2}-6 F-2\right)\right. \\
& \left.-d\left(F^{3}+7 F^{2}\right)\right] \\
C= & \frac{1}{2}\left[2 a\left(F^{3}+8 F^{2}-9 F-3\right)+b\left(F^{3}+8 F^{2}-6 F-4\right)\right. \\
& \left.-d\left(F^{3}+8 F^{2}\right)\right] \\
D= & -\frac{1}{6}\left[2 a\left(F^{3}+9 F^{2}-9 F\right)+b\left(F^{3}+9 F^{2}-6 F\right)\right. \\
& \left.-d\left(F^{3}+9 F^{2}+6\right)\right] .
\end{aligned}
$$

Using Eqs. (2) and (41), one can also find the expansion $(x>1)$ branch of the ASJEOS pressure:

$$
\begin{aligned}
& \text { ASJEOS: } \quad P(x) \\
& \qquad \begin{array}{l}
=\frac{e^{-F(x-1)}}{3 v_{0}}\left[\frac{3 A}{x^{6}}+\frac{2 B+A F}{x^{5}}+\frac{C+B F}{x^{4}}+\frac{C F}{x^{3}}+\frac{D F}{x^{2}}\right. \\
\left.+\frac{(A+B+C+D) F[F(x-1)-1]}{x^{2}}\right] \quad(x>1) .
\end{array}
\end{aligned}
$$

${ }^{1}$ W. B. Holzapfel, Rep. Prog. Phys. 59, 29 (1996).

${ }^{2}$ J. P. Perdew, H. Q. Tran, and E. D. Smith, Phys. Rev. B 42, 11627 (1990).

${ }^{3}$ F. D. Murnaghan, Proc. Natl. Acad. Sci. U.S.A. 30, 244 (1944).

${ }^{4}$ P. Vinet, J. Ferrante, J. R. Smith, and J. H. Rose, J. Phys. C 19, L467 (1986).

${ }^{5}$ P. Vinet, J. Ferrante, J. H. Rose, and J. R. Smith, J. Geophys. Res. 92, 9319 (1987).

${ }^{6}$ P. Vinet, J. H. Rose, J. Ferrante, and J. R. Smith, J. Phys.: Condens. Matter 1, 1941 (1989).

${ }^{7}$ J. C. Boettger and S. B. Trickey, Phys. Rev. B 53, 3007 (1996).

${ }^{8}$ J. Hama and K. Suito, J. Phys.: Condens. Matter 8, 67 (1996).

${ }^{9}$ R. E. Cohen, O. Gülseren, and R. J. Hemley, Am. Mineral. 85, 338 (2000).

${ }^{10}$ C. Zhang, S. Li, and Y. Teng, J. Phys. Chem. Solids 58, 835 (1997).

${ }^{11}$ L. Stixrude and R. E. Cohen, Science 267, 1972 (1995).

${ }^{12}$ J. P. Perdew, Phys. Lett. A 165, 79 (1992).

${ }^{13}$ F. Birch, Phys. Rev. 71, 809 (1947).

${ }^{14}$ F. Birch, J. Geophys. Res. 83, 1257 (1978).

${ }^{15}$ P. E. Van Camp, V. E. Van Doren, and J. T. Devreese, Phys. Rev. B 38, 12675 (1988).

${ }^{16}$ J. Meyer-ter-Vehn and W. Zittel, Phys. Rev. B 37, 8674 (1988).

${ }^{17}$ P. Söderlind and M. Ross, J. Phys.: Condens. Matter 12, 921 (2000).

${ }^{18}$ D. L. Novikov, M. I. Katsnelson, A. V. Trefilov, A. J. Freeman, N. E. Christenson, A. Svane, and C. O. Rodriguez, Phys. Rev. B 59, 4557 (1999).
${ }^{19}$ M. Ross, L. Yang, B. Dahling, and N. Winter, Z. Phys. Chem. (Munich) 184, 65 (1994).

${ }^{20}$ H. Maris and S. Balibar, Phys. Today 53 (2), 29 (2000).

${ }^{21}$ W. Yang, R. G. Parr, and L. Uytterhoeven, Phys. Chem. Miner. 15, 191 (1987).

${ }^{22}$ R. G. Pearson, J. Phys. Chem. 98, 1989 (1994).

${ }^{23}$ K. L. Sebastian, Chem. Phys. Lett. 231, 40 (1994).

${ }^{24}$ M. Hebbache, Solid State Commun. 113, 427 (2000).

${ }^{25}$ L. Pollack, J. P. Perdew, J. He, F. Nogueira, and C. Fiolhais, Phys. Rev. B 55, 15544 (1997).

${ }^{26}$ J. S. Dugdale and D. K. C. MacDonald, Phys. Rev. 89, 832 (1953).

${ }^{27}$ N. F. Mott and H. Jones, The Theory of the Properties of Metals and Alloys (Clarendon Press, Oxford, 1936).

${ }^{28}$ Y. Wang, D. Chen, and X. Zhang, Phys. Rev. Lett. 84, 3220 (2000).

${ }^{29}$ A. Kiejna, Prog. Surf. Sci. 61, 85 (1999).

${ }^{30}$ C. Fiolhais and J. P. Perdew, Phys. Rev. B 45, 6207 (1992).

${ }^{31}$ J. P. Perdew, Y. Wang, and E. Engel, Phys. Rev. Lett. 66, 508 (1991).

${ }^{32}$ J. P. Perdew, P. Ziesche, and C. Fiolhais, Phys. Rev. B 47, 16460 (1993).

${ }^{33}$ M. Seidl, J. P. Perdew, M. Brajczewska, and C. Fiolhais, J. Chem. Phys. 108, 8182 (1998).

${ }^{34}$ M. Payami, J. Chem. Phys. 111, 8344 (1999).

${ }^{35}$ I. Sarria, C. Henriques, C. Fiolhais, and J. M. Pitarke, Phys. Rev. B 62, 1699 (2000). 
${ }^{36}$ J. H. Rose and H. B. Shore, Phys. Rev. B 43, 11605 (1991).

${ }^{37}$ H. B. Shore and J. H. Rose, Phys. Rev. B 59, 10485 (1999).

${ }^{38}$ H. B. Shore and J. H. Rose, Phys. Rev. Lett. 66, 2519 (1991).

${ }^{39}$ V. Heine and D. Weaire, Solid State Phys. 24, 249 (1978). See p. 261.

${ }^{40}$ N. D. Lang and W. Kohn, Phys. Rev. B 1, 4555 (1970).

${ }^{41}$ N. D. Lang and W. Kohn, Phys. Rev. B 3, 1215 (1971).

${ }^{42}$ M. Manninen, Phys. Rev. B 34, 6886 (1986).

${ }^{43}$ N. W. Ashcroft and D. C. Langreth, Phys. Rev. 155, 682 (1967).

${ }^{44}$ D. M. Teter, G. V. Gibbs, M. B. Boisen, Jr., D. C. Allen, and M. P. Teter, Phys. Rev. B 52, 8064 (1995).

${ }^{45}$ A. K. McMahan and R. C. Albers, Phys. Rev. Lett. 49, 1198 (1982).

${ }^{46}$ C. Fiolhais, J. P. Perdew, S. Q. Armster, J. M. MacLaren, and M. Brajczewska, Phys. Rev. B 51, 14001 (1995); 53, 13 193(E) (1996).

${ }^{47}$ S. Kotochigova, Z. H. Levine, E. L. Shirley, M. D. Stiles, and C. W. Clark, Phys. Rev. A 55, 191 (1997).

${ }^{48}$ J. P. Perdew and A. Zunger, Phys. Rev. B 23, 5048 (1981).

${ }^{49}$ J. W. Mintmire, J. R. Sabin, and S. B. Trickey, Phys. Rev. B 26, 1743 (1982).

${ }^{50}$ J. C. Boettger and S. B. Trickey, Phys. Rev. B 32, 1356 (1985).

${ }^{51}$ J. C. Boettger, Int. J. Quantum Chem., Quantum Chem. Symp. 27, 147 (1993).

${ }^{52}$ U. Birkenheuer, J. C. Boettger, and N. Rösch, J. Chem. Phys. 100, 6826 (1994).

${ }^{53}$ U. Birkenheuer, Ph.D. thesis, TU München, 1994.

${ }^{54}$ J. C. Boettger, Int. J. Quantum Chem., Quantum Chem. Symp. 29, 197 (1995).

${ }^{55}$ J. C. Boettger, J. Phys.: Condens. Matter 11, 3237 (1999).

${ }^{56}$ J. P. Perdew, K. Burke, and M. Ernzerhof, Phys. Rev. Lett. 77, 3865 (1996); 78, 1396(E) (1997), and references therein.

${ }^{57}$ S. Kurth, J. P. Perdew, and P. Blaha, Int. J. Quantum Chem. 75, 889 (1999).
${ }^{58}$ M. Fuchs, M. Bockstedte, E. Pehlke, and M. Scheffler, Phys. Rev. B 57, 2134 (1998).

${ }^{59}$ D. M. Bylander and L. Kleinman, Phys. Rev. B 49, 1608 (1994).

${ }^{60}$ A. Zupan, K. Burke, M. Ernzerhof, and J. P. Perdew, J. Chem. Phys. 106, 10184 (1997).

${ }^{61}$ A. van de Walle and G. Ceder, Phys. Rev. B 59, 14992 (1999).

${ }^{62}$ J. C. Boettger and S. B. Trickey, Phys. Rev. B 29, 6425 (1984).

${ }^{63}$ F. Nogueira, C. Fiolhais, and J. P. Perdew, Phys. Rev. B 59, 2570 (1999).

${ }^{64}$ W. G. Zittel, J. Meyer-ter-Vehn, J. C. Boettger, and S. B. Trickey, J. Phys. F: Met. Phys. 15, L247 (1985).

${ }^{65}$ J. C. Boettger and R. C. Albers, Phys. Rev. B 39, 3010 (1989).

${ }^{66}$ J. B. Neaton and N. W. Ashcroft, (London) 400, 141 (1999). This article predicts a structural atom-pairing transition at $x=0.65$, which could mask the $2 s \rightarrow 2 p$ electronic transition in experiments.

${ }^{67}$ A. K. McMahan and J. A. Moriarty, Phys. Rev. B 27, 3235 (1983).

${ }^{68}$ J. D. Althoff, P. B. Allen, R. M. Wentzcovitch, and J. A. Moriarty, Phys. Rev. B 48, 13253 (1993).

${ }^{69}$ V. L. Moruzzi, J. F. Janak, and A. R. Williams, Calculated Electronic Properties of Metals (Pergamon, New York, 1978).

${ }^{70}$ N. A. W. Holzwarth and Y. Zeng, Phys. Rev. B 51, 13653 (1995).

${ }^{71}$ E. Wachowicz and A. Kiejna, Solid State Commun. 116, 17 (2000).

${ }^{72}$ J. M. Soler, Phys. Rev. Lett. 67, 3044 (1991).

${ }^{73}$ H. B. Shore and J. H. Rose, Phys. Rev. Lett. 67, 3045 (1991).

${ }^{74}$ C. Kittel, Introduction to Solid State Physics, 7th ed. (Wiley, New York, 1996).

${ }^{75}$ J. H. Rose, J. R. Smith, F. Guinea, and J. Ferrante, Phys. Rev. B 29, 2963 (1984).

${ }^{76}$ R. G. Greene, H. Luo, and A. L. Ruoff, Phys. Rev. Lett. 73, 2075 (1994). 